\title{
Aufsätze
}

Peter Becker

\section{Die Subsidiaritätsprüfung in Bundestag und Bundesrat - ein rechtliches oder ein politisches Instrument?*}

\section{Kurzfassung}

Der Vertrag von Lissabon hat die Rolle der nationalen Parlamente im politischen System der Europäischen Union aufgewertet. Von zentraler Bedeutung ist dabei die Prüfung der Einhaltung des Subsidiaritätsgrundsatzes durch die nationalen Parlamente im Rahmen eines sogenannten Frühwarnmechanismus. Dieses neue Instrumentarium der Mit- und Einwirkungsmöglichkeiten wird am Beispiel von Bundestag und Bundesrat analysiert. Trotz hoher Hürden haben die nationalen Parlamente im Sommer 2012 erstmals erfolgreich eine Subsidiaritätsrüge gegen die sogenannte Monti-II-Verordnung vorgebracht und damit den Stopp eines europäischen Gesetzgebungsvorhabens durchgesetzt. Das neue Verfahren befindet sich dennoch in der Erprobungsphase, seine Möglichkeiten und Grenzen werden noch ausgetestet. Einerseits öffnet die Subsidiaritätsprüfung neue politische Mitwirkungs- und Gestaltungskanäle für nationale Parlamentarier. Andererseits besteht die Gefahr, das Instrument durch eine politische Instrumentalisierung entlang nationaler Interessen für den europäischen Integrationsprozess zu entwerten.

* Der Autor dankt den Mitarbeiterinnen und Mitarbeitern der Europaausschüsse und -referate von Bundestag und Bundesrat, von Fraktionen und Länderverwaltungen für die Bereitschaft zu ausführlichen Hintergrundgesprächen, von denen dieser Text stark profitiert hat. Der Autor dankt auch den beiden anonymen Gutachtern für die konstruktiven Verbesserungsvorschläge und Anregungen an einer früheren Version des Beitrags. 


\section{Inhalt}

1. Einleitung 6

2. Die neue Rolle der nationalen Parlamente 7

3. Die bisherigen Erfahrungen und der erste erfolgreiche Anwendungsfall 13

4. Die Subsidiaritätsprüfung in Bundestag und Bundesrat 15

5. Die Subsidiaritätsrüge: Ein politisches oder rechtliches Instrument? 26

6. Fazit 32

\section{Einleitung}

Mit dem neuen Artikel 12 EUV widmet sich erstmals ein ganzer Vertragsartikel der Rolle der nationalen Parlamente in der Europäischen Union (EU). Demnach ist es die zentrale europapolitische Aufgabe der nationalen Parlamente, aktiv zur guten Arbeitsweise der Union beizutragen. Sie erhielten hierfür weitreichende Informations-, Mitwirkungs- und Kontrollrechte und sind erstmals direkt in den Fortgang des europäischen Integrationsprozesses eingebunden. Der Vertrag von Lissabon wurde deshalb auch bereits als „Vertrag der Parlamente“ (Brok/Selmayr 2008) bezeichnet.

Mit dem neuen Vertrag schien sich somit der Trend zur „Deparlamentarisierung“ der europäischen Politik umzukehren, der erstmals Mitte der neunziger Jahre des letzten Jahrhunderts konstatiert worden war (ausführlich bei Norton 1996, Börzel 2000 und O`Brennan/Raunio 2007). Dieser Prozess war gekennzeichnet von einer Marginalisierung und einem Kontrollverlust der nationalen Parlamente gegenüber ihren Exekutiven als Folge der zunehmenden und sich verdichtenden Europäisierung. Inzwischen habe sich aber nach einem intensiven parlamentarischen Lernprozess ,ein begrenzter Paradigmenwechsel hin zur 'Reparlamentarisierung' vollzogen“ (Kropp/Buzogány/Buche 2012: 115). Allerdings könnte sich auch dieser junge Parlamentarisierungstrend in der EU als kurze Zwischenetappe erweisen. Vor dem Hintergrund der aktuellen Eurozonen- und Staatsschuldenkrise besteht das Risiko, dass die nationalen Parlamente zu den Verlierern der europäischen Krisenbewältigungsversuche werden. Umso wichtiger wird es sein, die neuen Instrumente zur Stärkung der nationalen Parlamente im europäischen Integrationsprozess erfolgreich anzuwenden.

Unumstritten ist inzwischen, dass das zentrale Instrument des Vertrags von Lissabon die unmittelbare Einbindung der nationalen Parlamente in die europäische Gesetzgebungsarbeit in Form der Subsidiaritätsprüfung ist, der sogenannte Früh- 
warnmechanismus. Zumindest hat dieses Instrument des Vertrags von Lissabon insbesondere bei den wissenschaftlichen Beobachtern breite Aufmerksamkeit gefunden (Raunio 2009; Kropp/Buzogány/Buche 2012; Becker/Pintz 2012). Allerdings werden die Nutzbarkeit und die Effektivität dieses Instrumentariums sehr kritisch bewertet. So wird das Verfahren als ,stumpfes Schwert" bezeichnet (Höhlscheidt 2008: 264; Koch/Kullas 2010: 8 f.), es sei eher ein „Fremdkörper“ (Uerpmann-Wittzack 2009: 468) und ,im besten Falle ein Verzögerungsinstrument“ (Gröning-von Thüna 2010: 320).

Dennoch wurde im Sommer 2012 erstmals eine Subsidiaritätsrüge gegen den Vorschlag der Europäischen Kommission für eine „Verordnung über die Ausübung des Rechts auf Durchführung kollektiver Maßnahme im Kontext der Niederlassungs- und Dienstleistungsfreiheit“, die sogenannte Monti-II-Verordnung, erfolgreich angewandt. Diese erste erfolgreiche Subsidiaritätsrüge beendete ein Gesetzgebungsvorhaben, bevor der formelle Legislativprozess nach Artikel 294 AEUV überhaupt begonnen wurde. Diese faktische Wirkung des neuen Verfahrens dürfte weiter reichende Folgen für die künftige Nutzung des Frühwarnmechanismus sowohl durch die nationalen Parlamente als auch durch die Europäische Kommission haben.

Diese neue Form der Mit- und Einwirkungsmöglichkeiten der nationalen Parlamente auf die europäische Rechtsetzung soll am besonderen Beispiel von Bundestag und Bundesrat im Mittelpunkt dieses Beitrags stehen. Zunächst werden das Instrumentarium und dessen Anwendung kurz vorgestellt, bevor anschließend die Behandlung der bislang einzigen erfolgreichen Anwendung des Instruments bei der sogenannten Monti-II-Verordnung in Bundestag und Bundesrat näher untersucht wird. Abschließend soll die Nutzung der Subsidiaritätsprüfung als politisches oder rechtliches Instrument diskutiert werden.

\section{Die neue Rolle der nationalen Parlamente}

Formal zeigt sich die gestärkte Rolle der nationalen Parlamente darin, dass sie im Vertrag von Lissabon häufiger erwähnt werden als zuvor, nämlich 16 Mal. Zusätzlich konkretisieren zwei Protokolle des EU-Vertrags diese Mitwirkungs- und Kontrollrechte der nationalen Parlamente - das Protokoll über die Rolle der nationalen Parlamente in der Europäischen Union und das Protokoll über die Anwendung der Grundsätze der Subsidiarität und der Verhältnismäßigkeit.

Die Überwachung der Einhaltung des Subsidiaritätsgrundsatzes ist zweifellos das zentrale Instrument, an dem sich die gestärkte Rolle der nationalen Parlamente im europäischen Rechtsetzungsprozess am deutlichsten festmachen lässt. Die beiden 
Protokolle legen detailliert den Ablauf der Subsidiaritätsprüfung in vier Schritten fest (Becker/Kietz 2010):

1. Die EU-Kommission begründet ihre Legislativvorhaben mit Blick auf die Einhaltung der Grundsätze der Subsidiarität und der Verhältnismäßigkeit und übermittelt ihren Rechtsetzungsvorschlag gemeinsam mit dem Ergebnis der eigenen Subsidiaritätsprüfung direkt an die nationalen Parlamente. ${ }^{1}$

2. Binnen acht Wochen nach dem Zeitpunkt der Übermittlung eines Gesetzesentwurfs in allen Amtssprachen der Union können die nationalen Parlamente in einer begründeten Stellungnahme an die Präsidenten des Europäischen Parlaments, des Rates und der Kommission darlegen, weshalb der Entwurf ihres Erachtens nicht mit dem Subsidiaritätsprinzip vereinbar ist. In diesem Verfahren hat jedes Parlament zwei Stimmen. In Zwei-Kammer-Parlamenten hat dabei jede Kammer eine eigene Stimme und ein eigenes Recht zur Stellungnahme sowie zur Klageerhebung vor dem EuGH. Bei derzeitig 27 Parlamenten sind entsprechend 54 Stimmen verteilt. Über welche innerstaatlichen Verfahren Stellungnahmen beschlossen und Klagen erhoben werden können, ist im nationalen Recht und den Geschäftsordnungen der Kammern geregelt.

3. Erreicht die Anzahl begründeter Stellungnahmen mindestens ein Drittel der Gesamtzahl der den nationalen Parlamenten zugeteilten Stimmen, so muss der Entwurf durch den Urheber - in der Regel die Kommission - überprüft werden (,yellow card“). Bei derzeit 54 möglichen Stimmen sind also 18 Stimmen zur Auslösung dieses Verfahrens notwendig. ${ }^{2}$ Der Urheber des Gesetzentwurfs hat in der Folge drei Möglichkeiten: Er kann seinen Entwurf verwerfen und damit den Gesetzgebungsprozess bereits vor dessen Beginn beenden. Mit einer inhaltlichen Begründung kann er aber auch am Entwurf festhalten oder einen überarbeiteten Entwurf vorlegen und das Gesetzgebungsverfahren fortsetzen.

4. Erreicht die Anzahl der Stellungnahmen mindestens die Hälfte der den Parlamenten zugewiesenen Stimmen (,orange card“), also 27, und entscheidet sich die Kommission nach einer Überprüfung des Entwurfs, an dem unveränderten Vorschlag festzuhalten, werden die Stellungnahmen der Parlamente und die Begründung der Kommission Teil des ordentlichen Gesetzgebungsverfahrens und dem Europäischen Parlament und dem Rat vorgelegt. Diese müssen vor Abschluss der ersten Lesung prüfen und entscheiden, ob der Gesetzesvorschlag in

1 Der Löwenanteil der Vorschläge kommt von der EU-Kommission als Hauptinitiativorgan der EU; die Begründungspflicht bezieht sich aber auch auf Vorschläge der Mitgliedstaaten im Bereich der Justiz- und Innenpolitik.

2 Im Bereich der Innen- und Justizpolitik liegt diese Hürde niedriger; es genügt ein Viertel der zugewiesenen Stimmen, sprich 14. 
Einklang mit dem Subsidiaritätsprinzip steht. Sie können gegebenenfalls mit 55 Prozent der Stimmen im Rat bzw. einer Mehrheit im Europäischen Parlament entscheiden, den Gesetzgebungsprozess zu beenden.

Die nationalen Parlamente werden mit der vorgeschalteten Subsidiaritätsprüfung also zu Initiatoren eines eigenständigen Kontrollverfahrens, bevor eine Legislativinitiative formal auf die Tagesordnung des Rats gesetzt wird. Dieser Frühwarnmechanismus ist eine ,prä-legislative“ Möglichkeit der direkten Einwirkung auf den Willensbildungs- und Entscheidungsprozess innerhalb der Kommission und ihrer Dienststellen (Kiiver 2011). Die nationalen Parlamente haben sozusagen ein Vorprüfungsrecht (Cygan 2012) - allerdings mit einer deutlich eingeschränkten Wirkung, denn der Legislativvorschlag muss bei Beanstandungen allenfalls von der EU-Kommission überprüft werden. Darüber hinaus liegt die Hürde für die nationalen Parlamente zur Auslösung dieser Prüfverpflichtung an die Kommission sehr hoch und die Frist für die Abgabe einer begründeten Stellungnahme ist relativ knapp bemessen (Kiiver 2012). Um die kurze Zeitspanne für die Subsidiaritätsprüfung nicht noch weiter zu reduzieren, räumt die EU-Kommission den nationalen Parlamenten eine Unterbrechung des Fristablaufs von acht Wochen für den Sommermonat August ein. Diese Fristverlängerung gilt jedoch nicht in den sitzungsfreien Zeiten, über die Weihnachtsfeiertage oder in parlamentarischen Übergangszeiten, wenn ein Parlament neu gewählt wurde.

Eine Stellungnahme der nationalen Parlamente muss Gründe anführen, warum die Parlamentarier eine Verletzung des Subsidiaritätsprinzips rügen. Dennoch bleiben der Umfang der Prüfung, die Prüfschritte und die Kriterien der Prüfung unklar. Die bestehenden Mängel und Schwächen der mehrdeutigen Definition des Subsidiaritätsprinzips im europäischen Primärrecht seien auch mit dem Lissabonner Vertrag nicht behoben worden, so die Kritik (Koch/Kullas 2010). Es fehle dem Grundsatz weiterhin an Klarheit und Eindeutigkeit, was bereits in der Vergangenheit seine rechtliche Durchsetzbarkeit erschwert habe (Horsley 2012; Craig 2012).

Die EU-Organe sind jedoch schon seit dem Vertrag von Maastricht 1993 dazu verpflichtet, für die Einhaltung des Grundsatzes Sorge zu tragen und Stellung zu Subsidiaritätsfragen zu nehmen. ${ }^{3}$ Die Subsidiaritätskontrolle ist somit keine grundlegend neue Aufgabe, sondern erstreckt sich seit Einführung des Grundsatzes über den gesamten Verlauf der Rechtsetzungsverfahren. Jeder Legislativvorschlag muss unter dem Aspekt der Einhaltung des Prinzips begründet werden. Das Subsidiari-

3 Im sogenannten Subsidiaritätsprotokoll sowie in einer Interinstitutionellen Vereinbarung zur Subsidiarität zwischen Rat, Parlament und Kommission aus dem Jahr 1993 wurde diese Verpflichtung auf die Einhaltung des Subsidiaritätsgrundsatzes weiter präzisiert. 
tätsprotokoll des Lissabonner Vertrages präzisiert diese Begründungspflichten. Nunmehr muss jedem Legislativvorschlag ein „Subsidiaritätsvermerk“ mit detaillierten Angaben angefügt werden, der es den nationalen Parlamenten ermöglichen soll zu beurteilen, ob die Grundsätze der Subsidiarität und der Verhältnismäßigkeit geprüft und eingehalten wurden.

Die Kommission führt seit Einführung des Prinzips darüber hinaus umfangreiche Anhörungen durch, bevor sie einen Legislativvorschlag unterbreitet und legt jährlich über die Anwendung des Prinzips durch die EU-Institutionen einen Bericht vor (Europäische Kommission 2012 c). Die Prüfung findet vor allem im Rahmen der von den Kommissionsdienststellen organisierten Folgenabschätzungen zu geplanten Initiativen statt, deren Ergebnisse später auch in die Begründung der Rechtsetzungsvorschläge einfließen (Europäische Kommission 2012 a). Unter anderem enthält der Prüfleitfaden zur Durchführung der Folgenabschätzung einen konkreten Fragenkatalog zur Orientierung bei der Subsidiaritätsprüfung. Trotz einer relativ hohen Zahl (Europäische Kommission 2013 a) an Überarbeitungs- und Verbesserungsempfehlungen ${ }^{4}$ ist davon auszugehen, dass die Aufmerksamkeit und die Sensibilität innerhalb der Kommissionsdienststellen gegenüber den Subsidiaritätsbedenken aus den nationalen Parlamenten zugenommen haben (Craig 2012).

Auch Rat und Parlament müssen dem Subsidiaritätsgrundsatz während des gesamten Rechtsetzungsverfahrens Rechnung tragen. Zusätzlich kann die Konferenz der Ausschüsse für EU-Angelegenheiten der Parlamente der Europäischen Union (COSAC) zur Anwendung des Subsidiaritätsprinzips Stellung nehmen. Schließlich hat auch der Ausschuss der Regionen ein eigenes „Netz für Subsidiaritätskontrolle“ (Ausschuss der Regionen 2012) aufgebaut und hierfür ein eigenes umfangreiches Prüfraster entwickelt, mit dem die Rechtsgrundlage eines Legislativvorschlags, die ausreichende Beachtung des Subsidiaritäts- und des Verhältnismäßigkeitsgrundsatzes sowie der etwaig entstehende Finanz- und Verwaltungsaufwand abgefragt werden.

Dass neben den EU-Organen nun auch die nationalen Parlamente die Einhaltung des Prinzips kontrollieren können, ist als eine zusätzliche kompetenzrechtliche Si-

4 Im Jahr 2006 richtete die Kommission einen von ihren Dienststellen unabhängigen Ausschuss für Folgenabschätzung (Impact Assessment Board) ein, der eine intensivere Qualitätskontrolle der Legislativinitiativen gewährleisten sollte. Der Ausschuss prüft seit 2007 alle Folgenabschätzungen und empfiehlt in seinen Stellungnahmen an die Dienststellen der Kommission Nachbesserungen, unter anderem auch hinsichtlich der Bewertung der Subsidiarität und Verhältnismäßigkeit. Der Ausschuss kann auch eine erneute Vorlage der Folgenabschätzung zu einer zweiten Prüfung anfordern. 2012 nahm die Zahl der Bedenken des Ausschusses in Bezug auf eine ausreichende Berücksichtigung des Subsidiaritätsgrundsatzes zwar von 43 Prozent im Jahr 2011 auf 33 Prozent ab. Dennoch forderte der Ausschuss weitere Verbesserungen in Bezug auf das Subsidiaritätsprinzip. 
cherung zu verstehen. Bislang konzentrierte sich die Kontrolltätigkeit der nationalen Parlamente in der Europapolitik in erster Linie auf die Politik der eigenen Regierung. Deshalb haben die Parlamente ihre Aufgabe eher individuell und unkoordiniert wahrgenommen. Mit den neuen Beteiligungsmöglichkeiten und Kontrollrechten des Vertrags von Lissabon werden die nationalen Parlamente nun zu den wichtigsten „Subsidiaritätsprüfern“ in der EU; sie werden als die „Türwächter“ (Raunio 2011) und „Wachhunde“ (Cooper 2006) des Prinzips gesehen, denn entgegen den EUInstitutionen sind sie Kontrolleure in eigener Sache, die implizit auch die Einhaltung der Grenzen ihrer nationalstaatlichen Gesetzgebungsbefugnisse prüfen.

Allerdings dominierte bisher die Skepsis gegenüber der tatsächlichen Anwendung und Nutzbarkeit des Frühwarnmechanismus. Vier Gründe werden für die durchaus kritische Beurteilung vorgebracht:

1. Kritik wurde zunächst an der Ausgestaltung des Verfahrens selbst erhoben. Die Frist von lediglich acht Wochen, die den nationalen Parlamenten für die Prüfung eines Legislativvorschlags zur Verfügung steht, sei zu kurz bemessen (Cooper 2012: 449 f.; Koch/Kullas 2010: 9; Buzogány/Stuchlik 2012: 355). Diese Skepsis wurde dadurch bestätigt, dass noch im Jahr 2011 lediglich zehn Parlamente bzw. Kammern mit dem vorgegeben Zeitrahmen zufrieden waren (COSAC 2011: 35 ff.). Die kurze Prüffrist erschwere zudem die Möglichkeit zur interparlamentarischen Abstimmung, die allerdings erforderlich sei, um das vorgegebene Quorum von mindestens 18 Stimmen erreichen zu können.

2. Die Folgen einer Subsidiaritätsrüge, so die Kritik, seien zudem sehr limitiert; insbesondere gäbe es kein förmliches Veto der nationalen Parlamente, also keine „rote Karte“ (Cygan 2011: 487; Cooper 2012: 450). Die Kommission müsse lediglich ihren Legislativentwurf überdenken und es stehe ihr dann frei, wie sie mit den Einwänden und ihrem Vorschlag weiter verfahre.

3. Die begrenzte politische Nutzbarkeit des Instruments für die innenpolitische parlamentarische Auseinandersetzung reduziere auch das Interesse der nationalen Parlamentarier daran. Der geringe Anreiz für nationale Abgeordnete, sich mit der Prüfung und Kontrolle des Subsidiaritätsprinzips zu beschäftigen, werde noch dadurch verschärft, dass sich eine solche zeit- und arbeitsintensive Beschäftigung mit einem europapolitischen Thema nur sehr eingeschränkt in einem Wahlkampf in öffentliche Aufmerksamkeit, Unterstützung und damit in Wählerstimmen auszahlen dürfte (Raunio 2009; Cooper 2012). Insofern sollte auch das neue Instrument der Subsidiaritätsrüge die geringe Aufmerksamkeit für europapolitische Themen in der Innenpolitik nicht grundlegend verändern. Die begrenzte Bedeutung für die parlamentarische Arbeit nationaler Abgeordneter werde zudem beeinflusst von der jeweiligen Rolle des Parlaments im nationalen 
politischen System. Demnach scheinen Parlamente mit einer starken innenpolitischen Stellung eher zu einer strengen Kontrolle der europapolitischen Aktivitäten ihrer Exekutive zu neigen (Raunio 2009: 321; Raunio/Hix 2000).

4. Die Beobachter sind sich weitgehend einig, dass eine enge Abstimmung zwischen den Parlamenten erforderlich sei, um die Quoren zum Auslösen einer Subsidiaritätsrüge erreichen zu können. Dafür sei jedoch ein gemeinsames Verständnis über die Definition des Prüfmaßstabs „Subsidiarität“" erforderlich. Einer der Hauptgründe für die Zurückhaltung der nationalen Parlamente bei der Anwendung des Frühwarnmechanismus liege jedoch in einem unterschiedlichen Verständnis des Subsidiaritätsprinzips und dessen Prüfung. Die Parlamente legen ihren Prüfungen keine einheitliche Definition oder Prüfkriterien zugrunde; es gibt kein gemeinsames Prüfraster bzw. keine vereinbarte Schrittfolge der Prüfung. Dadurch variiert die Wahrnehmung, ob eine Subsidiaritätsverletzung vorliegt. Nicht in allen nationalen Parlamenten wird allerdings die Notwendigkeit gesehen, ein gemeinsames Verständnis zum Anwendungsbereich der Subsidiaritätsprüfung, konkrete gemeinsame Prüfleitlinien oder gar ein konkretes Prüfraster für die nationalen Parlamente zu entwickeln (COSAC 2012). ${ }^{5}$

Die Ausgestaltung und die Anwendung der Subsidiaritätsrüge bleibt demzufolge zunächst „sehr disparat" (Buzogány/Stuchlik 2012: 355) und der politische Mehrwert dieser vorgeschalteten Prüfung eines EU-Legislativvorschlags durch die nationalen Parlamente bleibt zweifelhaft. Denn im Verlauf des ordentlichen Gesetzgebungsverfahrens könnten die nationalen Parlamente im Rahmen ihrer innerstaatlichen Kontroll- und Mitwirkungsrechte ihre Regierung ohnehin zur Ablehnung des Vorschlags verpflichten. Die Subsidiaritätsprüfung ist im Kern also als zusätzliches defensives Kontrollinstrument der nationalen Parlamente ausgestaltet, das eine präventive Schutzwirkung entfalten soll. Es erlaubt eine Mitwirkung der nationalen Parlamente am europäischen Politikprozess nur in Bezug auf die enge Frage nach der angemessenen Ebene der sekundärrechtlichen Rechtsetzung und wird daher oft als Schutzschild zur Wahrung nationalstaatlicher Souveränitätsräume begriffen. Diese inhärente Defensivlogik legt den Fokus zwangsläufig auf „,negative interventions" (Cooper 2012: 450).

5 Das finnische Parlament wird in diesem Bericht dahingehend zitiert, dass es jeden Vorschlag für gemeinsame Vorgaben zur nationalparlamentarischen Subsidiaritätsprüfung ,strongly oppose“ (COSAC 2012). 


\section{Die bisherigen Erfahrungen und der erste erfolgreiche Anwendungsfall}

Die bisherigen Erfahrungen mit dem Frühwarnmechanismus des Vertrags von Lissabon erlauben angesichts der kurzen Spanne von lediglich drei Jahren allerdings nur begrenzte Rückschlüsse. Obgleich die Zahl der abgegebenen begründeten Stellungnahmen nur eine geringe Aussagekraft für die Nutzung der Subsidiaritätsrüge hat, zeichnet sich dennoch eine ansteigende Verwendung ab. Seit 2010 wurden EUweit insgesamt 225 begründete Stellungnahmen gegen einen Legislativvorschlag der EU von den nationalen Parlamenten abgegeben. Die Zahl der Subsidiaritätsrügen hat sich von 36 Rügen im Jahr 2010 (inklusive der begründeten Stellungnahmen, die allerdings erst nach der Acht-Wochen-Frist der EU-Kommission übermittelt wurden) im Jahr 2011 nahezu verdoppelt und im Jahr 2012 einen Stand von 70 begründeten Stellungnahmen erreicht. Zudem war die Streuung der Stellungnahmen der nationalen Parlamente immens (Europäische Kommission 2013 b). In der Regel entfiel bislang auf den gleichen Legislativvorschlag meist nur eine bis maximal vier Rügen. Die aktivsten Kammern waren in den Jahren 2010 bis 2012 der schwedische Riksdag, die luxemburgische Abgeordnetenkammer und die beiden polnischen Parlamentskammern (Europäische Kommission 2012 d).

Trotz dieser ernüchternden Zwischenbilanz waren die nationalen Parlamente erstmals am 22. Mai 2012 mit einer gemeinsamen Subsidiaritätsrüge erfolgreich. Mit zwölf begründeten Stellungnahmen und einem Gewicht von 19 Stimmen zur sogenannten Monti-II-Verordnung (Europäische Kommission 2012 b) wurde an diesem Tag das erforderliche Quorum für eine Rüge erreicht. Die Überlegungen zu einer gesonderten Verordnung über die Ausübung des Rechts auf kollektive Arbeitskampfmaßnahmen fand in der interessierten europäischen Öffentlichkeit eine breite Aufmerksamkeit. Insbesondere die europäischen Sozialpartner hatten frühzeitig das Vorhaben der Kommission als unangemessenen Eingriff in ihre Tarifhoheit kritisiert, sowohl gegenüber der Europäischen Kommission direkt als auch gegenüber den Abgeordneten im Europäischen Parlament sowie den Regierungen und den Parlamentariern in den Mitgliedstaaten. Die Kommission hatte die Vorstellung ihres Verordnungsentwurfs zunächst für Dezember 2011 angekündigt, dann aber wegen interner Abstimmungsprobleme auf Februar 2012 verschoben und schließlich am 21. März 2012 vorgelegt.

Die Kommission hatte in ihrem Verordnungsvorschlag (zusammen mit einem Vorschlag für eine Durchsetzungsrichtlinie) die Gleichrangigkeit der Ausübung sozialer Rechte und Kollektivmaßnahmen (wie Streiks) sowie der Niederlassungsund Dienstleistungsfreiheit betont. Die Verordnung solle die nationalen Rechtsvorschriften zum Streikrecht unberührt lassen; sie enthielt jedoch einen neuen Warn- 
mechanismus für Arbeitskämpfe mit gravierenden und grenzüberschreitenden Auswirkungen. Als Rechtsgrundlage stützte sich die Kommission auf die Abrundungsklausel in Artikel 352 AEUV. Der Vorschlag stand allerdings in einem Spannungsverhältnis zu Artikel 153 Abs. 5 AEUV, in dem das Streik- und das Aussperrungsrecht ausdrücklich der Regelungszuständigkeit der EU entzogen wird. Die Kommission berief sich deshalb in ihrer Begründung auf die Rechtsprechung des EuGH in den Fällen Viking (RS C-438/05 vom 11. Dezember 2007), Laval (C-341-/05 vom 18. Dezember 2007) und Rüffert (C-346/06 vom 3. April 2008), nach der das Arbeitskampfrecht nicht gänzlich dem Geltungsbereich des Unionsrechts entzogen

Tabelle 1: Begründete Stellungnahmen zur „Monti-II“-Verordnung (Deutscher Bundestag 2012) 6

\begin{tabular}{|c|c|c|c|}
\hline Parlament & Beschluss & Stimmen & $\begin{array}{l}\text { Begründung der } \\
\text { Rüge* }\end{array}$ \\
\hline Dänisches Parlament (Folketinget) & 3. Mai 2012 & 2 Stimmen & $1,2,3$ \\
\hline Schwedisches Parlament (Riksdag) & 10. Mai 2012 & 2 Stimmen & $1,2,3$ \\
\hline Polnischer Sejm & 11. Mai 2012 & 1 Stimme & 3 \\
\hline Luxemburgische Abgeordnetenkammer & 14. Mai 2012 & 2 Stimmen & 1,3 \\
\hline Finnisches Parlament (Eduskunta) & 15. Mai 2012 & 2 Stimmen & $1,3,4$ \\
\hline $\begin{array}{l}\text { Portugiesisches Parlament } \\
\text { (Assembleia da República) }\end{array}$ & 18. Mai 2012 & 2 Stimmen & 1,2 \\
\hline Belgisches Abgeordnetenhaus & 22. Mai 2012 & 1 Stimme & $1,2,4$ \\
\hline Französischer Senat & 22. Mai 2012 & 1 Stimme & 1,3 \\
\hline $\begin{array}{l}\text { Niederländisches Abgeordnetenhaus } \\
\text { (Tweede Kamer) }\end{array}$ & 22. Mai 2012 & 1 Stimme & $1,2,3$ \\
\hline $\begin{array}{l}\text { Maltesisches Abgeordnetenhaus } \\
\text { (Kamra tad-Deputati) }\end{array}$ & 22. Mai 2012 & 2 Stimmen & 3 \\
\hline Britisches Unterhaus (House of Commons) & 22. Mai 2012 & 1 Stimme & 3 \\
\hline Lettisches Parlament & 22. Mai 2012 & 2 Stimmen & $1,2,3,4$ \\
\hline Gesamt & & 19 Stimmen & \\
\hline
\end{tabular}

* Angegebene Gründe: 1. Fehlende oder falsche Rechtsgrundlage; 2. Keine Zuständigkeit der EU zur Regulierung des kollektiven Arbeitsrechts; 3. Kein rechtlicher oder politischer Mehrwert bzw. keine Notwendigkeit zum Erlass der Richtlinie; 4. Keine Berücksichtigung der Regeln des internationalen Arbeitsrechts.

Quelle: Eigene Zusammenstellung.

6 Die Stellungnahmen der nationalen Parlamente können über die IPEX-Datenbank abgerufen werden unter http://www.ipex.eu. 
sei. Der Vorschlag war insofern als Reaktion der Kommission auf die umstrittene und heftig kritisierte Rechtsprechung des Europäischen Gerichtshofs in diesen Streitfällen zu sehen, in denen der EuGH die Ausübung sozialer Grundrechte, wie des Streikrechts, gegenüber den garantierten Grundfreiheiten des Binnenmarktes abgewogen hatte (Mayer 2009).

Die nationalen Parlamente lehnten in ihren Subsidiaritätsrügen die gewählte Rechtsgrundlage ab. Eine europäische Harmonisierung des Arbeitskampfrechts sei eine deutliche Überschreitung der europäischen Kompetenzordnung. Die EU dürfe keinerlei Aspekte des Streikrechts regeln, da es sich ausschließlich um innerstaatliche Angelegenheiten handele. Darüber hinaus bemängelten die nationalen Parlamentarier, dass die Kommission nicht ausreichend dargelegt habe, wie durch den Vorschlag die entsprechenden Ziele der Verordnung erreicht werden können und wodurch ein europäischer Mehrwert geschaffen werden könne. Vielmehr enthalte der Vorschlag erneut unbestimmte Rechtsbegriffe, die insofern zu keiner Klärung der Rechtslage führten. Insbesondere bringe der Vorschlag keineswegs eine Klärung des umstrittenen Verhältnisses zwischen dem sozialen Grundrecht auf Streik und den Niederlassungs- und Dienstleistungsfreiheiten im europäischen Binnenmarkt. Am 12. September 2012 teilte die Europäische Kommission in einem Schreiben an die Präsidenten der nationalen Parlamente mit, dass sie ihren Verordnungsvorschlag nicht weiter verfolgen werde. ${ }^{7}$

Die nationalen Parlamente konnten also erstmals das neue Instrument für ihre Anliegen nutzen und ein europäisches Gesetzgebungsvorhaben stoppen. Ob allerdings dabei ein Verstoß gegen das Subsidiaritätsprinzip der entscheidende Beweggrund war, wurde zumindest von der Europäischen Kommission in ihrem Schreiben deutlich in Frage gestellt.

\section{Die Subsidiaritätsprüfung in Bundestag und Bundesrat}

Die beiden Kammern des deutschen Parlaments hatten sich bereits vor Inkrafttreten des Vertrags von Lissabon auf ihre neuen Mitwirkungs- und Kontrollrechte vorbereitet. Der Deutsche Bundestag überarbeitete seine internen, in seiner Geschäftsordnung geregelten Verfahren zur Behandlungen von Unionsdokumenten, um die Subsidiaritätsprüfung effektiv durchführen und sein Recht zur Einleitung einer Kla-

7 Die Antwortbriefe sind abrufbar unter http://ec.europa.eu/dgs/secretariat_general/relations/relation s_other/npo/letter_to_nal_parl_en.htm. 
ge wahrnehmen zu können. ${ }^{8}$ Zugleich baute der Bundestag seine personellen und finanziellen Kapazitäten zur Begleitung der europäischen Politik und zur faktischen und wirkungsvollen Wahrnehmung seiner neuen Rechte und Möglichkeiten deutlich aus. Zunächst wurde mit dem Referat „PA-1 Europa“ in der Abteilung der Sekretariate der Parlamentsausschüsse der Bundestagsverwaltung eine zentrale Institution geschaffen, um eine Priorisierung der EU-Dokumente und eine erste Subsidiaritätseinschätzung vorzunehmen. ${ }^{9}$ Im Januar 2013 baute die Bundestagsverwaltung ihre europapolitischen Ressourcen weiter aus und konzentrierte seine nunmehr sechs mit der Europapolitik befassten Referate in einer neugeschaffenen Unterabteilung PE (Parlament-Europa).

Für die Durchführung der eigentlichen Subsidiaritätsprüfung wurden allerdings keine gänzlich neuen Verfahren geschaffen. Vielmehr wurde die Prüfung in die bestehenden, allgemeinen Verfahren zur Behandlung von Unionsdokumenten integriert. Die Hauptzuständigkeit und die inhaltliche Verantwortung der Prüfung liegen demzufolge weiterhin bei den Fachausschüssen (§ 93 a der Geschäftsordnung des Bundestages). Allerdings wurde dem Europa-Ausschuss des Bundestages eine europapolitische Sonderrolle im Zusammenspiel mit den anderen Ausschüssen zugestanden.

Im Bundesrat ließen die Änderungen des Lissabonner Vertrags die internen Regelungen und Abläufe zur Mitwirkung an der deutschen Europapolitik und dem Politikzyklus auf europäischer Ebene weitgehend unverändert. Im Unterschied zum Bundestag sah der Bundesrat keine Notwendigkeit, seine Geschäftsordnung im Hinblick auf die Möglichkeiten und Erfordernisse der Subsidiaritätskontrolle abzuändern. Lediglich das bestehende Ausschussverfahren erfuhr kleinere technische Verfahrensanpassungen. Zur weiteren Konkretisierung des Prüfverfahrens legte im Januar 2013 eine länderoffene Arbeitsgruppe „Subsidiarität“ des EU-Ausschusses des Bundesrates nach fast zwei Jahre andauernden Verhandlungen eine interne Handreichung zur Handhabung der Subsidiaritätsrüge vor. Ein besonderes Ziel des zunächst als Leitfaden bezeichneten Dokuments ist es, die sehr unterschiedlichen

8 Bereits im Rahmen seiner Ratifizierung des Lissabonner Vertrages im Jahr 2008 hatte der Bundestag über eine Änderung seiner Geschäftsordnung seine internen Verfahren angepasst, um die Subsidiaritätsprüfung in das Regelverfahren zur Behandlung von EU-Vorhaben einzubetten. Letzte Änderungen wurden im Juli 2010 verabschiedet; vgl. Bekanntmachung zur Änderung der Geschäftsordnung des Deutschen Bundestages vom 16.7.2010, BGB1. I S. 1041 (Nr. 39).

9 Diesem Referat war auch die neugeschaffene Repräsentanz des Deutschen Bundestages bei der EU in Brüssel zugeordnet. 
internen Prüfverfahren in den Ländern anzugleichen. ${ }^{10}$ Mit der rechtlich und politisch nicht verbindlichen Handreichung des Bundesrates soll nun eine einheitliche Hilfestellung für die Subsidiaritätsprüfung in den Ländern und eine gemeinsame Grundlage unter gleichzeitiger Wahrung der divergierenden Rechtsansichten der einzelnen Länder geschaffen werden.

Zusätzlich hat der Bundesrat 2013 auch eine Vertretung in den regelmäßigen Abstimmungs- und Informationsgesprächsrunden der Vertretungen der nationalen Parlamente in Brüssel geschaffen, dem „Network of National Parliament Representatives“ bzw. den „Monday Morning Meetings“. Der Mitarbeiter des Sekretariats des EU-Ausschusses des Bundesrats pendelt zwischen Brüssel und Berlin und hat die Aufgabe, frühzeitig und umfassend über etwaige Subsidiaritätsbedenken und -prüfungen in anderen nationalen Parlamenten zu berichten und umgekehrt die Bedenken im Länderkreis gegenüber einzelnen Legislativvorhaben der EU in die Beratungen in Brüssel einzubringen. Somit ist der Bundesrat erstmals direkt in Brüssel mit einem eigenen Repräsentanten vertreten.

Derzeit verläuft das Prüfverfahren im Rahmen des Frühwarnmechanismus in Bundestag und Bundesrat in vier Schritten:

(1) Vorprüfung durch die Bundesregierung: Am Beginn steht jeweils die Übermittlung des Gesetzgebungsvorschlags durch die Europäische Kommission und entsprechend dem EUZBBG ${ }^{11}$ und dem EUZBLG ${ }^{12}$ durch die Bundesregierung. Die Bundesregierung ist dazu verpflichtet, den übermittelten EU-Dokumenten einen Berichtsbogen hinzuzufügen, mit dem Bundestag und Bundesrat in knapper Form über Inhalt und Bedeutung des EU-Vorhabens und über die weiteren Verfahrensschritte informiert werden. Bereits dieser Berichtsbogen enthält eine gesonderte Rubrik, in der die Bundesregierung ihre Bewertung zur Vereinbarkeit und Berücksichtigung des EU-Vorhabens mit den Grundsätzen der Subsidiarität und der Verhältnismäßigkeit darlegt.

Handelt es sich um einen europäischen Legislativvorschlag, übermittelt die Bundesregierung zusätzlich zu ihrem Berichtsbogen eine weitere Stellungnahme, die sogenannte „umfassende Bewertung“(UBW). Diese UBW muss, so schreibt es das EUZBBG vor, zwei Wochen nach der Überweisung an die Ausschüsse des Bun-

10 Im Kreis der Länder blieben einige grundsätzliche Fragen allerdings umstritten, zu denen die Handreichung insofern keine abschließende Lösung formuliert, wie beispielsweise, ob Fragen der Rechtsgrundlage oder des Verhältnismäßigkeitsgrundsatzes Bestandteile der Subsidiaritätsprüfung sein sollen.

11 Gesetz über die Zusammenarbeit von Bundesregierung und Deutschem Bundestag in Angelegenheiten der Europäischen Union.

12 Gesetz über die Zusammenarbeit von Bund und Ländern in Angelegenheiten der Europäischen Union. 
destages vorliegen. Die inhaltliche Prüfung durch das jeweilige federführende Bundesministerium geht dabei regelmäßig deutlich über die erste Bewertung des Berichtsbogens hinaus. Hierfür hat die Bundesregierung in ihrer gemeinsamen Geschäftsordnung (GGO) ein eigenes Prüfraster und Verfahrensgrundsätze erstellt. ${ }^{13}$ In diesen Bewertungen der EU-Dokumente legt die Bundesregierung ihre möglichen Subsidiaritätsbedenken gegen einen europäischen Gesetzgebungsvorschlag dem Bundestag und dem Bundesrat vor. Für die Subsidiaritätsprüfung in Bundestag und Bundesrat legt die Bundesregierung also eine Art Vorprüfung vor (Schröder 2012: 254).

(2) Eigene Prüfung durch die Verwaltungen von Bundestag und Ländern: Auf der Grundlage dieser ersten Bewertungen der Bundesregierung nehmen dann in einem zweiten Schritt Bundestag und Bundesrat ihre internen Einschätzungen vor, ob ein Unionsdokument Anlass zu Subsidiaritätsbedenken geben könnte. Für den Bundestag war bisher das zentrale Europareferat PA 1 hierfür zuständig. Seit Januar 2013 wird diese Aufgabe je nach Politikbereich und Federführung auf zwei Referate - PE 2 und PE 3 - aufgeteilt. Die beiden Referate haben die Aufgabe, aus der Masse von jährlich ca. 25.000 EU-Dokumenten, die beim Bundestag eingehen, eine erste Einstufung in beratungsrelevante und nicht beratungsrelevante Vorhaben vorzunehmen. Zwischen 500 und 600 Vorhaben werden so den Ausschüssen des Bundestages zur Beratung zugewiesen (Schröder 2012: 270). Eine Schätzung der Bundestagsverwaltung geht von 20 bis 25 umfassenden Prüfungen pro Jahr aus. Im Rahmen dieser inhaltlichen Priorisierung machen die zuständigen Referenten auch Angaben hinsichtlich der Subsidiaritätsfrage und verweisen auf die Fristen für eine Prüfung im Ausschuss und im Plenum des Bundestages und insbesondere auf das Ende der Acht-Wochen-Frist, innerhalb der eine begründete Stellungnahme nach Brüssel übermittelt werden muss. Zusätzlich wird im Zuge der Erstellung der Sachstandsberichte durch die Verwaltung zu einzelnen Dossiers und den europapolitischen Vorausschauen frühzeitig auf mögliche Subsidiaritätsbedenken hingewiesen. Die politischen Fraktionen - das heißt die Fraktionsobleute in den einzelnen Ausschüssen - treffen die abschließende politische Entscheidung darüber, ob eine Prüfung im zuständigen Ausschuss durchgeführt wird, die am Ende gegebenenfalls in eine Subsidiaritätsrüge des Bundestages münden kann (Gröning-von Thüna 2010: $320 \mathrm{ff}$.).

Die besondere Stärke der Subsidiaritätskontrolle durch den Bundesrat liegt sicherlich in der direkten Einbeziehung von 16 Landesverwaltungen in diese Prüfung. Nicht der Bundesrat oder dessen Verwaltung führen de facto die inhaltliche und 
kompetenzrechtliche Prüfung von EU-Dokumenten durch, sondern die Exekutiven der Länder. Jede Landesverwaltung hat inzwischen ein eigenes Prüfraster entwickelt, um einerseits die Prüfung und die eigene Positionierung im Bundesrat vorzubereiten, aber auch um andererseits die eigenen Landesparlamente über eigene Subsidiaritätsbedenken schnell informieren zu können.

Das Sekretariat des EU-Ausschusses im Bundesrat stellt die eingehenden Entwürfe für europäische Gesetzgebungsvorhaben in einer gesonderten Frühwarnliste zusammen ${ }^{14}$ und weist dabei auch auf den Fristablauf für eine Subsidiaritätsrüge und auf die innerhalb dieser Frist zu erreichende Plenartagung des Bundesrates hin. Seit Inkrafttreten des Vertrags von Lissabon am 1. Dezember 2009 sind insgesamt 381 dieser Frühwarndokumente beim Sekretariat des EU-Ausschusses des Bundesrats eingegangen, von denen 334 als Bundesrats-Drucksachen umgedruckt und beraten wurden.

(3) Inhaltliche Prüfung in den Ausschüssen von Bundestag und Bundesrat: In einem dritten Verfahrensschritt werden dann in den Fachausschüssen des Bundestages im Zuge der üblichen Beratungen von Unionsdokumenten grundsätzlich auch die Einhaltung der Grundsätze der Subsidiarität und Verhältnismäßigkeit geprüft. Halten die Fachausschüsse eine Rüge für angebracht, geben sie eine entsprechende Beschlussempfehlung ab. Dem EU-Ausschuss werden dennoch besondere Mitwirkungsmöglichkeiten eingeräumt. Die Fachausschüsse müssen den EU-Ausschuss unverzüglich darüber informieren, sobald sie eine Rüge in Betracht ziehen. Dem Europa-Ausschuss muss die Gelegenheit zur Abgabe einer eigenen Stellungnahme ermöglicht werden, die dann zusammen mit der Rügeempfehlung des Fachausschusses vom Plenum beraten werden muss ( 93 a GO). Zudem kann der EU-Ausschuss das Plenum befassen, falls er im Unterschied zu den Fachausschüssen eigene Subsidiaritätsbedenken hinsichtlich eines Vorhabens hegt.

Die eigentliche Subsidiaritätsprüfung obliegt dennoch im Regelfall dem federführenden Ausschuss des Bundestages. Da sich die Fachausschüsse mit dem komplizierten Instrumentarium der Subsidiaritätsprüfung und der Rüge häufig nur sehr sporadisch befassen, scheint die Sensibilität für die Beachtung des Subsidiaritätsgrundsatzes und die potenzielle politische Nutzbarkeit des Instrumentariums sehr unterschiedlich ausgeprägt zu sein. Das inzwischen eingespielte Verfahren des EUAusschusses, zunächst nur das Fristende bis zur Abgabe einer Subsidiaritätsrüge zur Kenntnis zu nehmen, erlaubt es den Europapolitkern zu einem späteren Zeitpunkt, das EU-Dokument nochmals inhaltlich eingehend unter subsidiaritätsrecht-

14 Die Frühwarnliste wird in der EUDISYS-Datenbank des Bundesrates (EU-Dokumenten-Informations-System) eingestellt und regelmäßig aktualisiert. 
lichen Gesichtspunkten zu prüfen. Die erste formelle Befassung bietet also ausreichenden verfahrensrechtlichen und zeitlichen Spielraum, um andere Fachausschüsse des Bundestages zu sensibilisieren und die Abstimmung mit ihnen zu suchen sowie um die Einschätzungen in anderen nationalen Parlamenten über das Büro des Bundestages und die Vertreter der Bundestagsfraktionen in Brüssel einzuholen. Damit behält sich der EU-Ausschuss die Wiedervorlage des EU-Dokuments vor, auch wenn die anderen Fachausschüsse mit einer frühzeitigen Kenntnisnahme des Dokuments den Vorgang bereits für sich förmlich abgeschlossen haben und somit eine vertiefte Prüfung nicht mehr möglich wäre. Diese Praxis des EU-Ausschusses wertet die Sonderstellung gegenüber den übrigen Bundestagsausschüssen zusätzlich auf und kann die Konkurrenzsituation weiter verstärken.

Für den Bundesrat übernehmen die Fachministerien in den Landesregierungen die inhaltliche und subsidiaritätsrechtliche Prüfung der vom Sekretariat des EUAusschusses des Bundesrates zusammengestellten Frühwarndokumente. Die Behandlung der Dokumente in den Ausschüssen des Bundesrats erfolgt dann nach dem üblichen Verfahren in den sogenannten Ausschusswochen vor den monatlichen Plenartagungen der Länderkammer. Für die Formulierung des Beschlussentwurfs wird in den Ausschussberatungen zwischen der förmlichen Subsidiaritätsrüge und der inhaltlichen Stellungnahme zu einem EU-Legislativvorschlag unterschieden. ${ }^{15}$ In der Regel sollen Subsidiaritätsrüge und inhaltliche Stellungnahme zusammen von den Ausschüssen erarbeitet werden. Sollte dies nicht möglich sein, kann eine inhaltliche Stellungnahme auch später in den Fachausschüssen des Bundesrats erstellt werden, das heißt nachdem die Rüge fristgerecht nach Brüssel übermittelt worden ist. Federführend für die Erarbeitung einer Stellungnahme oder einer Rüge im Bundesrat bleibt der EU-Ausschuss, der die Empfehlungen der Fachausschüsse in einer Synthese zu einer Beschlussvorlage für die Befassung durch das Plenum des Bundesrats zusammenfasst. ${ }^{16}$

15 Die stehende Formulierung für eine Subsidiaritätsrüge des Bundesrats lautet: „Der Ausschuss empfiehlt dem Bundesrat zu der Vorlage gemäß Artikel 12 Buchstabe b EUV wie folgt Stellung zu nehmen $[\ldots]$ ". Bei inhaltlichen Stellungnahmen ohne eine förmliche Rüge nimmt der Bundesrat auf seine Rechte aus den $\S \S 3$ und 5 des EUZBLG Bezug.

16 Für den Fall abweichender Stellungnahmen der Fachausschüsse, also wenn ein oder mehrere Ausschüsse die Abgabe einer Subsidiaritätsrüge empfehlen, während andere Ausschüsse dies nicht tun, noch nicht Stellung genommen oder eine Stellungnahme vertagt haben, kann das Plenum mit der vorgeschlagenen Subsidiaritätsstellungnahme befasst werden. Nach den eingespielten Verfahren des Bundesrats wäre eine solche, zwischen allen Ausschüssen noch nicht abschließend ausgehandelte Stellungnahme für eine Plenarbefassung noch nicht beratungsreif. Um allerdings die AchtWochen-Frist einhalten zu können, sollen die Bedenken eines Fachausschusses Vorrang haben vor der formalen Befassung aller Ausschüsse. 
(4) Politische Entscheidung zur Abgabe einer Rüge: Grundsätzlich soll die letztinstanzliche Entscheidung über die Erhebung einer Rüge im jeweiligen Plenum von Bundestag und Bundesrat gefällt werden, wobei stets mit Mehrheit abgestimmt wird. ${ }^{17}$ Der Bundestag kann aber auf Antrag einer Fraktion oder von fünf Prozent der Abgeordneten auch den EU-Ausschuss ermächtigen, über eine Subsidiaritätsrüge anstelle des Bundestagsplenums allein zu entscheiden ( $\$ 93$ c GO). ${ }^{18}$ Dennoch ist die Beschlussfassung im Plenum die Regel. ${ }^{19}$

Der Deutsche Bundestag sprach bislang drei Subsidiaritätsrügen aus, die noch keine endgültigen Rückschlüsse auf die Handhabung erlauben. Der Bundesrat verabschiedete deutlich mehr begründete Stellungnahmen als der Bundestag, nämlich bislang elf förmliche Rügen. Jedoch fanden zwölf Anträge zur Erhebung einer Subsidiaritätsrüge nicht die erforderliche Mehrheit in der Länderkammer. Zur Wahrung der Acht-Wochen-Frist kann der Bundesrat auch auf sein gesondertes Instrument der Europakammer zurückgreifen, die ihrerseits auch in einem schriftlichen Umlaufverfahren beschließen kann. Die Rüge des Bundesrates vom 26. März 2010 zur mitgliedstaatlichen Initiative für eine europarechtliche Schutzanordnung war europaweit die erste Subsidiaritätsrüge überhaupt nach Inkrafttreten des Frühwarnmechanismus.

Die bisherigen Erfahrungen in Bundestag und Bundesrat mit dem neuen Instrument der Subsidiaritätsrüge zeigen, dass die zunächst geäußerte Kritik an dem Verfahren und die Skepsis gegenüber der Nutzbarkeit sich nur zum Teil als zutreffend erwiesen haben. Bundestag und Bundesrat waren durchaus in der Lage, die Acht-WochenFrist für die Abgabe einer begründeten Stellungnahme einzuhalten. ${ }^{20}$

17 Im Gegensatz zur Erhebung einer Subsidiaritätsklage vor dem EuGH ist die Rüge also nicht als Minderheitenrecht ausgestaltet.

18 Bereits im Rahmen der allgemeinen Mitwirkung des Bundestages an der deutschen Europapolitik erlaubt Art. 45 GG dem Plenum, den EU-Ausschuss zu ermächtigen, das Recht des Bundestages auf Stellungnahme gegenüber der Regierung nach Art. 23 GG wahrzunehmen.

19 Eine Übertragung der Beschlussfassung würde die ohnehin kritisch beäugte Sonderstellung des EU-Ausschusses gegenüber den anderen Fachausschüssen weiter verstärken und auch deutlich sichtbar machen.

20 Diese Frist beginnt nach Vorlage der letzten Übersetzung in eine der offiziellen Amtssprachen der Europäischen Union (Artikel 6, Protokoll Nr. 2). Da aber die Sprachfassungen in den häufiger gesprochenen Amtssprachen (insbesondere Englisch, Französisch und Deutsch) deutlich früher zugestellt werden als die Übersetzungen in anderen Sprachen, ist die Frist für Bundestag und Bundesrat de facto länger als acht Wochen. 


\begin{tabular}{|c|c|c|c|c|c|c|c|c|c|}
\hline 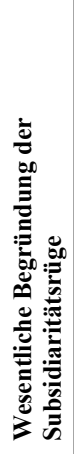 & & 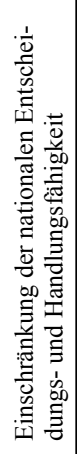 & 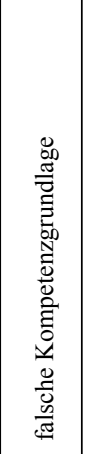 & 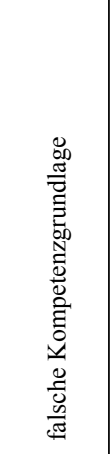 & & 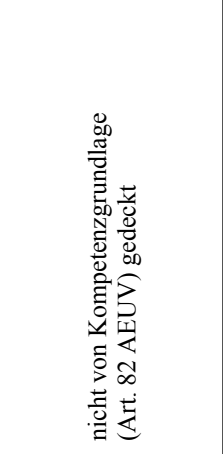 & 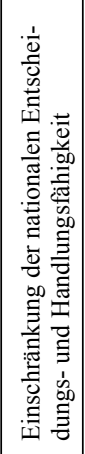 & 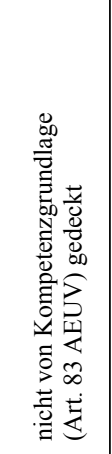 & 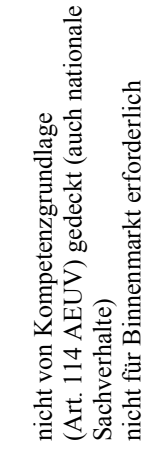 \\
\hline 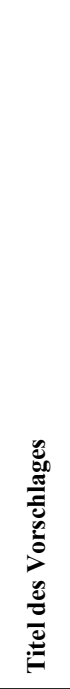 & & 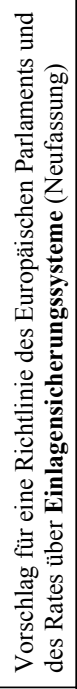 & 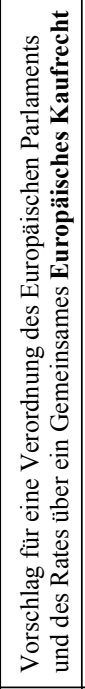 & 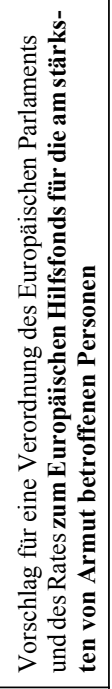 & & 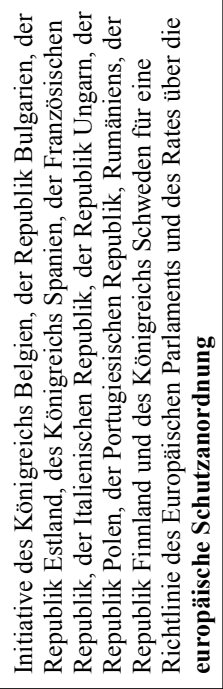 & 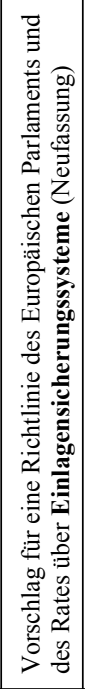 & 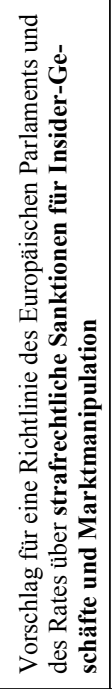 & 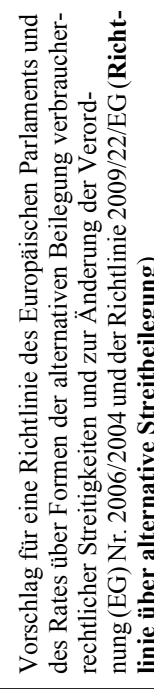 \\
\hline$\dot{\Delta}$ & & స్ స్ & $\begin{array}{l}\stackrel{8}{\infty} \\
\stackrel{\infty}{\Sigma} \\
=\end{array}$ & 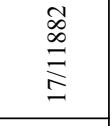 & & $\stackrel{\circ}{\stackrel{\gamma}{\gamma}}$ & 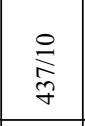 & $\begin{array}{l}\bar{J} \\
\text { J } \\
\end{array}$ & 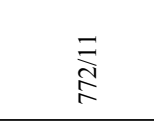 \\
\hline & & 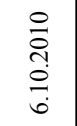 & 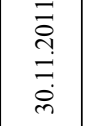 & 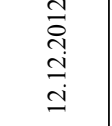 & & 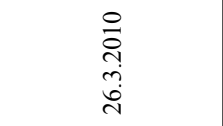 & $\begin{array}{l}\stackrel{\circ}{\grave{\lambda}} \\
\text { aे } \\
\dot{\sim}\end{array}$ & 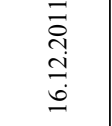 & 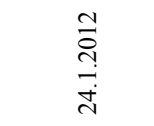 \\
\hline 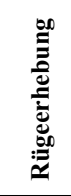 & 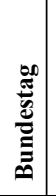 & 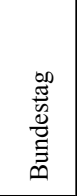 & 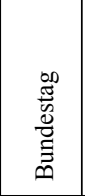 & 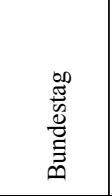 & 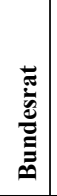 & 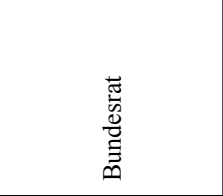 & 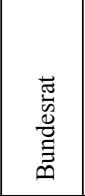 & 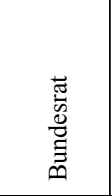 & 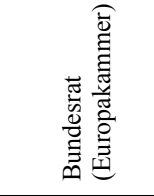 \\
\hline
\end{tabular}


Die Subsidiaritätsprüfung in Bundestag und Bundesrat

\begin{tabular}{|c|c|c|c|c|c|c|}
\hline 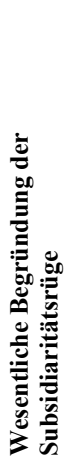 & 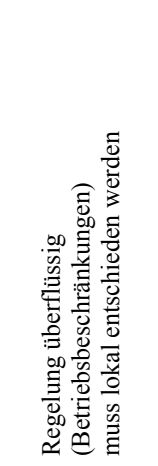 & 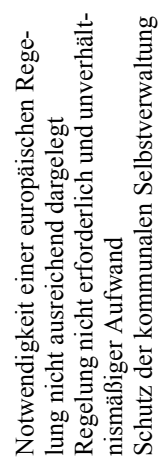 & 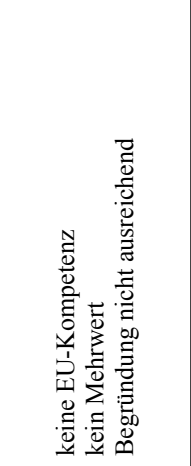 & 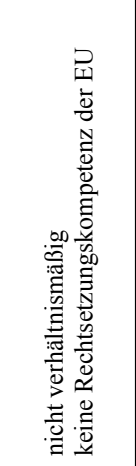 & 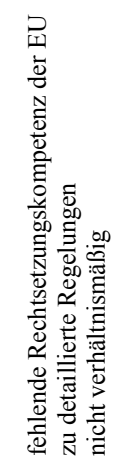 & 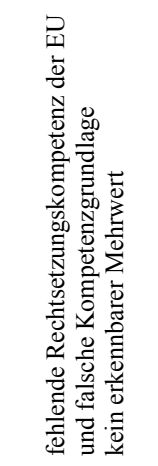 \\
\hline 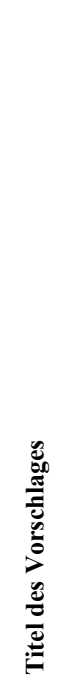 & 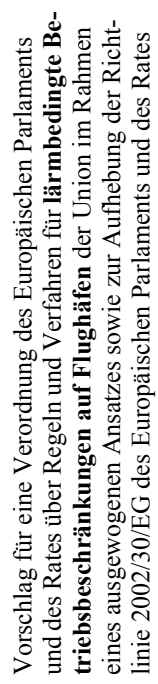 & 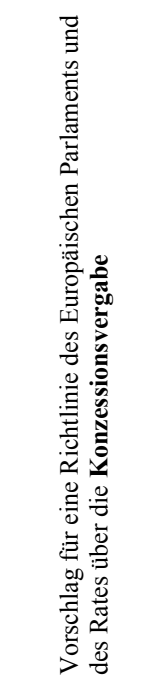 & 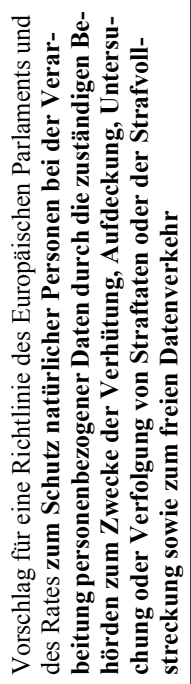 & 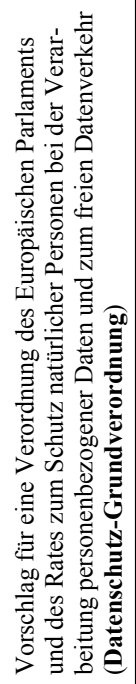 & 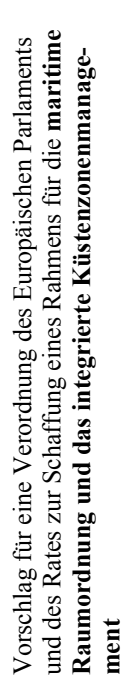 & 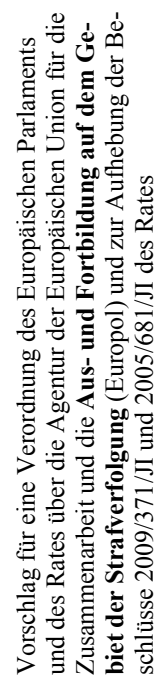 \\
\hline$\dot{\dot{\omega}}$ & $\underset{\frac{1}{2}}{\stackrel{\Xi}{2}}$ & $\underset{\infty}{\stackrel{\vec{J}}{\vec{J}}}$ & $\underset{n}{\stackrel{I}{I}}$ & $\underset{\sim}{\stackrel{N}{\pi}}$ & $\stackrel{m}{m}$ & 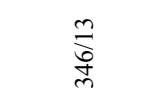 \\
\hline $\begin{array}{l}E \\
\stackrel{E}{E} \\
\text { O }\end{array}$ & $\begin{array}{l}\stackrel{N}{\Delta} \\
\stackrel{\sim}{N} \\
\end{array}$ & 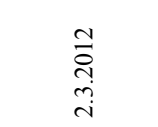 & 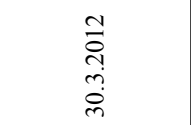 & 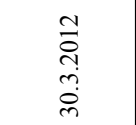 & 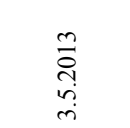 & 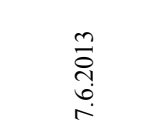 \\
\hline 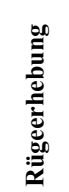 & 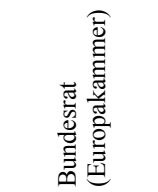 & 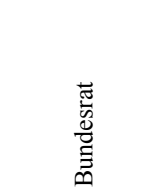 & 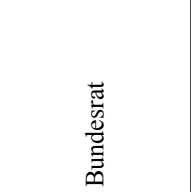 & 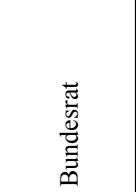 & 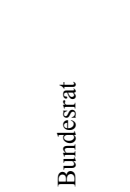 & 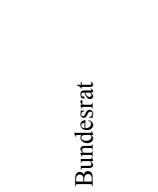 \\
\hline
\end{tabular}


Angesichts der begrenzten personellen und finanziellen Ressourcen, die zur Priorisierung und Prüfung der EU-Dokumente zur Verfügung stehen, und der Masse an Unionsdokumenten, die zur inhaltlichen Prüfung an Bundestag und Bundesrat übermittelt werden, wird allerdings nicht jedes EU-Vorhaben einer ausführlichen Subsidiaritätsprüfung unterzogen werden können. Von besonderer Bedeutung ist daher das Ergebnis der Vorprüfung durch die Bundesregierung in ihren Umfassenden Bewertungen. Die Bewertung eines europäischen Gesetzgebungsvorschlags durch das jeweilige Bundesministerium sensibilisiert die Verwaltung des Bundestages und die Sekretariate der Fachausschüsse für mögliche Subsidiaritätsprobleme. Die Bundesregierung filtert aus der Vielzahl der Legislativvorschläge die aus ihrer Sicht besonders sensiblen Fälle aus, die einer besonderen Aufmerksamkeit des Parlaments bedürfen sollten. Sie kann so auch den Tenor der parlamentarischen Prüfung vorzeichnen. Während jedoch im Europa-Ausschuss des Bundestages die Abgeordneten nicht auf diese Vorprüfungen durch die Bundesministerien Bezug nehmen, folgen die Ministerialbeamten der Länder in den Ausschüssen des Bundesrates häufiger dem Votum ihrer Bundeskollegen und nehmen in ihren Ausschussberatungen durchaus darauf Bezug. Für die Abgeordneten des Bundestages scheinen hingegen die Stellungnahmen starker Interessengruppen und Verbände für ihre Positionierung von ebenso wichtiger Bedeutung zu sein.

Der erste erfolgreiche Anwendungsfall des Frühwarnmechanismus hat auch die Befürchtung widerlegt, die Hürde zur Auslösung der gelben Karte sei unerreichbar hoch. Allerdings verwundert es, dass die erste erfolgreiche Anwendung des Instruments der Subsidiaritätsrüge durch die nationalen Parlamente ohne Beteiligung von Bundestag und Bundesrat zustande kam. Für die Bundesregierung hatte das federführende Ministerium für Arbeit und Soziales zwar seine Skepsis mit Blick auf die gewählte Kompetenzgrundlage des Verordnungsvorschlags geäußert. Dennoch kam die Bundesregierung in ihrer Umfassenden Bewertung vom 18. Mai $2012 \mathrm{zu}$ dem Gesamtfazit, dass der Vorschlag ,nicht von vornherein mit dem Grundsatz der Subsidiarität unvereinbar"s sei. Diese Schlussfolgerung bestimmte offenkundig auch die Bewertung im Bundesrat und den federführenden Fachressorts in den Ländern.

Die Länder erkannten im Zuge ihrer ersten Beratungen in den Ausschüssen ebenfalls keine Beeinträchtigung des Subsidiaritätsgrundsatzes. Der Bundesrat verabschiedete deshalb auch keine Subsidiaritätsrüge. Erst nachdem sich herausgestellt hatte, dass europaweit das notwendige Quorum für eine ,gelbe Karte“ von 18 Stimmen von den nationalen Parlamenten erreicht werden konnte, erarbeiteten die Länder in einer zweiten Beratungsrunde in den Ausschüssen eine inhaltliche Stellungnahme, in der sie den Verordnungsvorschlag ablehnten. Dieser Beschlussvorschlag wurde vom Plenum am 15. Juni 2012 angenommen (Bundesrat 2012). In seiner 
kompetenzrechtlichen und inhaltlichen Bewertung unterscheidet sich dieser auf die Mitwirkungsrechte des EUZBLG gestützte Beschluss des Bundesrates nicht von den formellen Subsidiaritätsrügen der anderen nationalen Parlamente.

Im Bundestag hatte sich der federführende Ausschuss für Arbeit und Soziales am 25. April und am 23. Mai 2012 mit der Monti-II-Verordnung befasst, ohne allerdings einen Beschluss zu verabschieden. Im April legte die Bundesregierung ihre Bewertung vor; im Anschluss wurde die Behandlung des EU-Dokuments ebenso wie die Abstimmung über einen von den Fraktionen der SPD und Bündnis 90/Die Grünen eingebrachten Entschließungsantrag vertagt. Der mitberatende Ausschuss für Wirtschaft und Technologie hatte bereits am 9. Mai 2012 das EU-Dokument ohne eine inhaltliche Stellungnahme zur Kenntnis genommen und damit seine Befassung abgeschlossen. Der ebenfalls mitberatende Europaausschuss des Bundestages hatte seinerseits am 9. Mai die Subsidiaritätsfrist zur Kenntnis genommen und am 19. Mai 2012 eine weitere, inhaltliche Behandlung des EU-Dokuments vertagt.

\section{Tabelle 3: Verfahrensschritte bei der Behandlung der Monti-II-Verordnung}

\begin{tabular}{|c|c|c|}
\hline \multicolumn{3}{|c|}{$\begin{array}{l}\text { Europäische Kommission: } \\
\text { Vorschlag für eine Verordnung des Rates über die Ausübung des Rechts auf Durchführung kollektiver Maßnahmen } \\
\text { im Kontext der Niederlassungs- und der Dienstleistungsfreiheit, KOM (2012) } 130 \text { endg., Brüssel, den 21.3.2012 }\end{array}$} \\
\hline \multicolumn{3}{|c|}{$\begin{array}{l}\text { Bundesregierung: } \\
\text { Bundesministerium für Arbeit und Soziales (federführend), Bundesministerium für Wirtschaft und Technologie, } \\
\text { Bundesministerium der Justiz, Auswärtiges Amt } \\
\text { Berichtsbogen vom 3. April 2012 } \\
\text { Umfassende Bewertung vom 18. Mai } 2012\end{array}$} \\
\hline & Bundestag & Bundesrat \\
\hline Eingang & 27.3.2012 & 23.3.2012 \\
\hline $\begin{array}{l}\text { Ausschuss- } \\
\text { befassung }\end{array}$ & $\begin{array}{l}\text { Arbeit und Soziales (federführend): } \\
\text { Vertagung; } \\
\text { Entschließungsantrag von SPD und Bündnis 90/ } \\
\text { Die Grünen wurde nicht abgestimmt } \\
\text { Wirtschaft und Technologie: } \\
\text { 9.5.2012 Kenntnisnahme } \\
\text { EU-Angelegenheiten: } \\
\text { 9.5.2012 Vertagung } \\
\text { 23.5.2012 Vertagung }\end{array}$ & $\begin{array}{l}23 .-27.4 .2012 \\
\text { Beratung in den Ausschüssen: } \\
\text { Arbeit und Sozialpolitik } \\
\text { Recht } \\
\text { Wirtschaft } \\
\text { Europa (federführend) }\end{array}$ \\
\hline Fristablauf & 22.5 .2012 & 22.5.2012 \\
\hline $\begin{array}{l}\text { Erneute Befas- } \\
\text { sung Ausschüsse }\end{array}$ & & 29.05.-1.6.2012 \\
\hline Befassung Plenum & & $\begin{array}{l}\text { 15.6.2012 } \\
\text { Beschluss Drs. 157/12 }\end{array}$ \\
\hline
\end{tabular}


Die Mehrheitsfraktionen im Bundestag hatten also die Behandlung des Verordnungsvorschlags bis zum Ablauf der Subsidiaritätsfrist vertagt und somit eine inhaltliche Debatte über den EU-Vorschlag umgangen - und dies, obgleich zumindest im Europaausschuss die Möglichkeit zur erstmaligen erfolgreichen Anwendung der Subsidiaritätsrüge durch die nationalen Parlamente durchaus erkannt worden war.

Während im Bundesrat die Nichtbeteiligung an der Subsidiaritätsrüge eher als Unfall interpretiert wird, dominierten im Bundestag hingegen parteitaktische Erwägungen. Im federführenden Arbeits- und Sozialausschuss waren die Regierungsfraktionen offenkundig nicht bereit, den europaweit, insbesondere von den europäischen Gewerkschaften ${ }^{21}$ getragenen Protest gegen das Vorhaben der EU-Kommission mit der Zustimmung zum Antrag der Oppositionsparteien zu verstärken. Im EU-Ausschuss kam noch die Erwägung hinzu, dass die Europapolitiker diese politische Positionierung der Fachpolitiker nicht überstimmen wollten. Die (fehlende) Debatte zum Monti-II-Vorschlag der EU-Kommission im Bundestag war insofern eine Folge von drei bislang ungeklärten Spannungslinien:

1. zwischen Regierungs- und Oppositionsfraktionen mit ihren unterschiedlichen politischen Zielen bei der Subsidiaritätsprüfung;

2. zwischen dem federführenden Fachausschuss und dem EU-Ausschuss hinsichtlich der unausgesprochenen, aber dennoch bestehenden Konkurrenzsituation bei der Behandlung von EU-Vorlagen;

3. zwischen der fachpolitisch-inhaltlichen Bewertung eines EU-Dokuments und der Nutzung der Subsidiaritätsrüge als kompetenzrechtliches Instrument zur Abgrenzung gegenüber der europäischen Rechtsetzung.

\section{Die Subsidiaritätsrüge: Ein politisches oder rechtliches Instrument?}

Die nationalen Parlamente werden mit dem neuen Instrumentarium des Vertrags von Lissabon zu Initiatoren eines eigenständigen Subsidiaritäts-Kontrollverfahrens, bevor eine Legislativinitiative formal auf die Tagesordnung des Rats gesetzt wird. Dieser neue Mit- und Einwirkungskanal der nationalen Parlamente an der Fortentwicklung des europäischen Integrationsprozesses wirft die grundsätzliche Frage auf, ob der neugeschaffene Frühwarnmechanismus vornehmlich als kompetenzrechtliches Kontrollinstrument von den nationalen Parlamenten genutzt werden sollte, mit dem der Initiator eines europäischen Legislativvorhabens auf eine potenzielle Überschreitung der im Vertrag von Lissabon fixierten Kompetenzverteilung aufmerksam gemacht werden soll, oder ob die nationalen Parlamente nicht viel

21 Deutlich ablehnende Stellungnahmen des Europäischen Gewerkschaftsbundes und des DGB lagen seit April 2012 vor. 
eher ihre Stellungnahmen und Rügen als Möglichkeit zur proaktiven Politikgestaltung und zur frühzeitigen Einflussnahme auf die Inhalte der europäischen Gesetzgebung nutzen sollten. Regelmäßig wird deshalb zwischen politischer Wünschbarkeit und kompetenzrechtlicher Richtigkeit der europäischen Gesetzgebung abzuwägen sein.

Schon in der Debatte des Bundesrates über die erste begründete Stellungnahme (Bundesrat 2010) gegen den Vorschlag der Europäischen Kommission zur Einführung einer europäischen Schutzanordnung im März 2010 zeigte sich, wie schwierig und umstritten diese Abwägung ist. Obgleich alle Mitglieder des Bundesrates das Ziel des Richtlinienvorschlags teilten und den Inhalt unterstützen, verabschiedete die Mehrheit des Bundesrates die europaweit erste Subsidiaritätsrüge mit dem Hinweis auf die falsche Rechtsgrundlage der Initiative. ${ }^{22}$ Die Mehrheitsmeinung argumentierte, dass die Prüfung der Kompetenzübertragung eine erforderliche Vorfrage sei, um in einem zweiten Schritt anschließend die Frage nach der rechtmäßigen Kompetenzausübung beantworten zu können (so auch Buschmann/Daiber 2011). Die Subsidiaritätsprüfung durch die nationalen Parlamente sei insofern zunächst eine juristische Frage nach der Rechtmäßigkeit eines europäischen Legislativvorschlags (Suhr 2010: 23 f.). Mit dem Hinweis auf die ursprüngliche Debatte im europäischen Verfassungskonvent (Europäischer Konvent 2003: 145) widersprachen allerdings die Kritiker diesem Ansatz. Das Subsidiaritätsprinzip sei in erster Linie von politischer Natur und unterliege deshalb einer politischen Kontrolle durch die nationalen Parlamente - die Subsidiaritätskontrolle sei keine Kompetenzkontrolle (Mayer 2010: 16).

Dennoch wird die nationalparlamentarische Subsidiaritätskontrolle nur eingeschränkt als Instrument zur proaktiven, politikgestaltenden Einbindung der nationalen Parlamente wahrgenommen. Vielmehr entfaltet das „Frühwarnsystem“ zunächst und vor allem eine aufschiebende Wirkung auf den europäischen Gesetzgebungsprozess - insbesondere nachdem das Instrument bei der Monti-II-Richtlinie erstmals seine Bremswirkung unter Beweis stellen konnte. Eine solche Fokussierung auf den vornehmlich defensiven und aufschiebenden Charakter wird jedoch von einzelnen Beobachtern (Uerpmann-Wittzack 2009) und Praktikern aus dem Bundestag in Frage gestellt (Gröning-von Thüna 2010) oder sogar abgelehnt (Semmler 2010). Die Subsidiaritätskontrolle sei als „Einmischungsgebot“ zu verstehen, das neue Kanäle der parteipolitischen Netzwerkbildung innerhalb der euro-

22 Die Mindermeinung kommt in der Erklärung der rheinland-pfälzischen Staatsministerin Margit Conrad zum Ausdruck. Siehe Bundesrat, Plenarprotokoll zur 868. Sitzung am 26. März 2010, Anlage 15, 114. 
päischen Parteifamilien eröffne. Die Subsidiaritätskontrolle solle deshalb „flexibel““ als Teil des europäischen political bargaining genutzt werden und die politische Aussagekraft des Subsidiaritätsprinzips nutzen (ebd.). Parlamente seien keine Gerichte, die die Einhaltung der europäischen Kompetenzordnung und des Subsidiaritätsgrundsatzes entlang rechtlicher Kriterien prüfen sollten. Vielmehr sei die Subsidiaritätsrüge ein politisches Instrument, das den Mehrheitsfraktionen in den mitgliedstaatlichen Parlamenten die Möglichkeit der offensiven und effektiven Nutzung zur konstruktiven Mitgestaltung in der Europapolitik eröffne. Das Ergebnis einer Subsidiaritätsprüfung werde insofern ,,je nach parteipolitischer Prägung unterschiedlich ausfallen“ (Gröning-von Thüna 2010: 322).

Gerade vor diesem Hintergrund ist die Frage, an wen die begründete Stellungnahme eines nationalen Parlamentes de facto adressiert ist, von besonderer politischer Bedeutung für die Nutzung des Frühwarnmechanismus. Zweifellos richtet sich eine begründete Stellungnahme im Rahmen einer Subsidiaritätsrüge zunächst an den Verfasser des Legislativvorschlags und damit formal an die EU und ihre Organe. Allerdings besteht die vorrangige Aufgabe nationaler Parlamente darin, die Politik der eigenen Regierung auf europäischer Ebene zu kontrollieren. Für Bundestag und Bundesrat besteht darüber hinaus aus dem Lissabon-Urteil des Bundesverfassungsgerichts vom Juni 2009 (BVerfG, 2 BvE 2/08 u. a. vom 30.6.2009) sogar eine besondere Integrationsverantwortung, die die Beachtung des Grundsatzes der Subsidiarität einschließt. Demnach besteht eine besondere Verpflichtung zur Subsidiaritätsprüfung für Bundestag und Bundesrat: „Das Lissabon-Urteil hat die Bedeutung der Subsidiaritätskontrolle daher insofern verändert, als zu ihrer europarechtlichen Verankerung und dem wohlverstandenen Eigeninteresse des Bundestages an der Wahrung seiner politischen Gestaltungsmöglichkeiten nun noch unzweifelhaft eine gleichgerichtete verfassungsrechtliche Pflicht hinzugetreten ist." (Lorz 2010: 3) Diese Form der Integrationsverantwortung bedeutet, dass die Bundesregierung ein zusätzlicher Adressat der Subsidiaritätskontrolle von Bundestag und Bundesrat ist.

Obgleich die Prüfung der Kompetenzordnung und der Subsidiarität zunächst keine Frage der nationalstaatlichen Verfassungsordnung ist, sondern eine europarechtliche Aufgabe, verfügen die nationalen Parlamente in erster Linie gegenüber ihren nationalstaatlichen Exekutiven im Zuge der innerstaatlichen parlamentarischen Kontrolle und Mitwirkung der Europapolitik über wirksame Sanktionsinstrumente. Vor allem gegenüber der Bundesregierung können die beiden Kammern ihre kontrollierende Rolle mit dem Instrument der Subsidiaritätsprüfung ausüben. Insofern besteht ein Missverhältnis zwischen den eigentlichen Prüfinhalten und der europarechtlichen Funktion der Subsidiaritätskontrolle einerseits sowie dem Adressaten 
der Prüfergebnisse und der politischen Wirksamkeit auf der anderen Seite. So kann die Verknüpfung von defensiv-präventivem Charakter des Frühwarnmechanismus und parteipolitischer Polarisierung bei der Anwendung der Subsidiaritätsprüfung zu einer stärkeren nationalen Betrachtungsweise und Instrumentalisierung des Instrumentariums führen. Unter solchen Rahmenbedingungen wäre es vorrangig die nationale Öffentlichkeit, die sich entlang parteipolitischer Familien in Befürworter oder Gegner eines Legislativvorschlags gruppieren würde. Im Zweifel werden die kompetenzrechtlichen Kriterien des Subsidiaritätsprinzips zurücktreten und die Verteidigung nationalstaatlicher Souveränitätsansprüche wird dominieren, auch wenn die europäische Ebene besser in der Lage sein sollte, eine Regulierungslücke zu füllen. Für die nationalen Parlamentarier rückt die Frage nach der europarechtlichen Kompetenzordnung und der richtigen Entscheidungsebene zur Ausübung einer übertragenen Kompetenz in den Hintergrund und die politische Wünschbarkeit bzw. Ablehnung eines Vorschlags wird zum entscheidenden Kriterium. Nicht die Frage „Ist die europäische Entscheidungsebene besser in der Lage, eine Maßnahme umzusetzen und ein politisches Ziel zu erreichen?" bestimmt die Entscheidung der nationalen Parlamente, sondern die Frage „Ist diese europäische Rechtsetzung gewollt?". Eine solche politische Instrumentalisierung des Instruments der Subsidiaritätsrüge zeigte sich bereits bei der ersten begründeten Stellungnahme des Deutschen Bundestages zum Richtlinienvorschlag für ein europäisches Einlagensicherungssystem. Bundestag und Bundesrat wurden massiv von den Dachverbändern von Sparkassen und Volksbanken bedrängt, das Instrument der Rüge zur Ablehnung des Vorschlags zu nutzen, obgleich die Bundesregierung bei ihrer umfassenden Bewertung und ihrem internen Berichtsbogen einen Verstoß gegen das Subsidiaritätsprinzip nicht erkennen konnte und allenfalls nicht ausschließen wollte.

Darüber hinaus kann die Subsidiaritätsrüge auch als verhandlungstaktisches Instrument im Zusammenspiel von Regierung und nationalem Parlament gegenüber den EU-Organen genutzt werden. Im Unterschied zu einer Regierung, die aus politischen oder aus taktischen Erwägungen nicht offen gegen einen Gesetzgebungsvorschlag der EU auftreten will, den Vorschlag aber dennoch ablehnt, weil die Regierung nicht zur Abgabe nationaler Zuständigkeiten bereit ist, können sich die Mehrheitsfraktionen in einem nationalen Parlament in Abstimmung mit ihrer Regierung sehr viel leichter und ohne verhandlungstaktische Rücksichtnahme gegen einen Vorschlag aus Brüssel positionieren.

Die Ausgestaltung der Subsidiaritätsrüge als Recht der parlamentarischen Mehrheit kann demzufolge die Nutzbarkeit des Instruments in der politischen Auseinandersetzung innerhalb eines Parlaments verringern. Die Prüfung europäischer Gesetzgebung ist jedoch gerade für die jeweilige parlamentarische Opposition ein 
wichtiges Instrument zur Kontrolle und Mitwirkung an der nationalen Europapolitik. Für die Mehrheitsfraktionen, die ohnehin die nationale Europapolitik über ihren direkteren Zugang zur Regierung effektiver beeinflussen können, ist die Anwendung der Subsidiaritätsrüge hingegen ,ein Balanceakt zwischen Kontrolle und Unterstützung“" (Buzogány/Stuchlik 2012: 355) der eigenen Regierung. Es ist selten, dass die Mehrheitsfraktionen zu einer grundsätzlich anderen Bewertung und Haltung gegenüber einer Initiative der EU-Kommission kommen, als die Regierung zumal die Regierungen üblicherweise vor der Vorlage eines Legislativentwurfs intensiv von den Kommissionsdienststellen konsultiert werden. Sollte dieser Fall gegensätzlicher politischer Bewertungen eines EU-Gesetzgebungsvorhabens zwischen Regierung und Regierungsfraktionen allerdings auftreten, so eröffnet das Instrument der Subsidiaritätsrüge den Parlamentariern einen neuen Weg, der eigenen Regierung konkrete Grenzen für ihre Verhandlungsführung in Brüssel aufzuzeigen - unabhängig davon, ob europaweit das Quorum der gelben Karte des Frühwarnmechanismus erreicht werden kann. Eigentlicher Adressat der Bedenken der Parlamentsmehrheit ist auch in diesem Fall zunächst die eigene Regierung und erst danach die EU.

Wenn aber das Instrument der europäischen Subsidiaritätsrüge in erster Linie für die innenpolitische Profilierung und die parteipolitische Polarisierung genutzt wird, gewinnt die Subsidiaritätskontrolle zwar an Attraktivität für die nationalen Parlamente. Deren Stellungnahmen müssen in der Folge aber nicht unbedingt von der EU-Kommission und dem Europäischen Parlament als abgewogener inhaltlicher Beitrag zur besseren Legislativtätigkeit der EU aufgenommen werden, insbesondere, wenn sie zur Verhinderung europäischer Rechtsetzung eingesetzt werden. Der Einfluss der Positionierungen nationaler Parlamente auf die Meinungsbildung der EU-Organe könnte sich reduzieren. Das Instrument würde seinen politischen Wert zur proaktiven und politikgestaltenden Mitwirkung an der europäischen Gesetzgebung verlieren und endgültig auf seine politische Nutzbarkeit als defensives Verhinderungs- oder zumindest Verzögerungsinstrument einer qualifizierten Minderheit nationaler Parlamentskammern reduziert werden.

Allerdings öffnet sich der neue politische Einfluss- und Mitwirkungskanal auf die Legislativtätigkeit der EU den nationalen Parlamenten nur dann, wenn sie koordiniert und geschlossen agieren. Dies wird nur möglich werden, wenn die Parlamente ihre potenzielle Macht erkennen und ihre Stimme als Kollektiv effektiv organisieren und vor allem dann, wenn die Mehrheitsfraktionen in den nationalen Parlamenten gewillt sein werden, dieses Instrument auch konsequent zu nutzen. „Der Dreh- und Angelpunkt für die Stärkung der politischen Bedeutung der Subsidiaritätsrüge ist daher die Abstimmung zwischen den nationalen Parlamenten." (Semmler 2010: 
533) Hierfür ist dann jedoch ein sehr ähnliches Verständnis des Subsidiaritätsgrundsatzes und dessen Anwendung im Zuge des Frühwarnmechanismus in den nationalen Parlamenten erforderlich.

Ein europaweites Subsidiaritätsverständnis zur politisch-gestaltenden, proaktiven Nutzbarkeit des Instrumentariums wird sich langfristig erst dann entwickeln können, wenn die Europäisierung der nationalen Parlamente auch zu einer Aufwertung europäischer Politik in der informellen nationalen parlamentarischen Themenhierarchie führen wird. Erst wenn mit dem Engagement der Abgeordneten für eine subsidiäre europäische Gesetzgebung auch eine erhöhte innerstaatliche mediale Aufmerksamkeit und damit die Chance zur parlamentarischen und innerparteilichen Profilierung verbunden sein wird, erst dann könnte das Instrument seine volle Attraktivität für die nationalen Parlamente entfalten und damit zu einem wirkungsvollen europapolitischen Gestaltungswerkzeug werden.

Die weiterhin bestehende Mehrdeutigkeit des Subsidiaritätsprinzips ist aber gerade eine besondere Schwäche. Der Grundsatz ist zwar als ein rechtliches Prinzip im europäischen Vertragsrecht verankert, aber es fehlen dennoch eine klare Definition und eindeutige rechtliche Prüfkriterien. Die Formulierung im Vertrag von Lissabon beinhaltet eine Reihe unbestimmter Rechtsbegriffe, für die der Europäische Gerichtshof bislang noch keine abschließende inhaltliche Definition vorgelegt hat. Diese Ambivalenz und Interpretationsoffenheit macht eine einheitliche rechtlich-materielle Prüfung des Prinzips in den nationalen Parlamenten schwierig.

Andererseits führt das Problem zu geringer oder fehlender Politisierung zu verringerter Aufmerksamkeit für den Frühwarnmechanismus. Wenn also einige Parlamente und Parlamentarier in erster Linie zu inhaltlichen Fragen eines europäischen Legislativvorschlags Stellung nehmen und den Meinungsbildungsprozess beeinflussen wollen, so verringert eine solche pragmatisch-sachliche Nutzung verbunden mit den formalen Beschränkungen des Instrumentariums dessen Attraktivität für nationale Parlamentarier. Auf europäischer Ebene wäre gerade eine solche, an pragmatischen, europarechtlichen Kriterien orientierte Vorgehensweise von Vorteil und würden dem Wunsch der nationalen Parlamentarier nach politisch-gestaltender Mitwirkung eher entsprechen.

Auch die Reaktion der Kommission auf die erfolgreiche Rüge gegen die Monti-IIVerordnung verstärkte die Nutzbarkeit der Subsidiaritätsrüge entlang politischer Wünschbarkeiten und nationaler Befindlichkeiten. Die Kommission sah keineswegs den Grundsatz der Subsidiarität verletzt, sondern erkannte die realpolitischen Konsequenzen der Rüge. Angesichts der politischen Ablehnung in 12 von 27 nationalen Parlamenten und des Erfordernisses der Einstimmigkeit zur Verabschiedung der Verordnung im Ministerrat war eine Umsetzung der Verordnung nicht 
mehr realistisch. Die Kommission beugte sich dem politischen Widerstand, aber nicht dem Subsidiaritätsargument.

\section{Fazit}

Vor diesem Hintergrund reicht die Anwendung des neuen Instruments der Subsidiaritätsrüge weit über die Frage nach Prüfungsumfang und Adressat der Prüfung hinaus. Vielmehr geht es um die Nutzung des Frühwarnmechanismus als rein defensiv-reaktives, europarechtliches Verhinderungswerkzeug zur Kontrolle der europäischen Kompetenzordnung, oder als Politik gestaltendes, proaktives Instrument der nationalen Parlamente zur Mitwirkung am europäischen Entscheidungsprozess.

Unabhängig davon, mit welcher Prämisse und wie sorgsam die einzelnen Kammern der nationalen Parlamente ihre Rolle als Überwachungsinstitutionen des Subsidiaritätsprinzips wahrnehmen, bleibt festzuhalten, dass sich die Rolle der nationalen Parlamente im Rahmen der europäischen Integrationspolitik verändern wird. Diese Veränderungsprozesse sollen hier zu vier thesenartigen Prognosen kondensiert werden:

1. Die Möglichkeit zur unmittelbaren Mit- und Einwirkung auf die europäische Willensbildung und den europäischen Politikprozess wird mittelfristig die Aufmerksamkeit in den nationalen Parlamenten für die europäische Politik potenziell erhöhen. Das Interesse der nationalen Parlamentarier an dem Instrumentarium wird dann weiter ansteigen, wenn sie sich wirksamer, sichtbarer und unmittelbarer an der Europapolitik beteiligen können.

2. Diese zunehmende Attraktivität der Europapolitik für die nationalen Parlamente wird zunächst die innerstaatliche und innenpolitische Diskussion über europapolitische Themen und die europäische Gesetzgebung aufwerten. Dabei wird nicht die europarechtliche Frage im Vordergrund der national-parlamentarischen Betrachtung stehen, sondern die Instrumentalisierbarkeit in der innenpolitischen Auseinandersetzung zwischen Regierungs- und Oppositionsfraktionen. Adressaten dieser europapolitischen Subsidiaritätsdebatten in den nationalen Parlamenten werden allerdings nicht die Legislativorgane der Europäischen Union sein, sondern eher die nationale Europapolitik der eigenen Regierung.

3. Das neue Instrumentarium der Subsidiaritätskontrolle kann deshalb zu innenpolitischen Gewichtsverschiebungen zwischen Regierung und Parlament führen. Zwar werden die nationalen Parlamente in ihrer Rolle als Hüter des Subsidiaritätsprinzips gestärkt, die nunmehr in die bislang autonom von den Regierungen vorgenommenen Subsidiaritätsprüfungen einbezogen werden müssen. Aber gerade vor dem Hintergrund der innenpolitischen Dominanz der national- 
parlamentarischen Subsidiaritätsdebatten werden die Bewertungen der Regierungen auch die Analysen der Mehrheitsfraktionen und damit letztlich der Parlamente vorprägen. Nicht die europarechtliche Frage nach der angemessenen Entscheidungsebene wird die Positionierung der nationalen Parlamente bestimmen, sondern die innenpolitische Auseinandersetzung zwischen Regierungsund Oppositionsfraktionen. Die Regierungsfraktionen werden sich in der national-parlamentarischen Debatte gedrängt sehen, die eigene Regierung zu stützen und deren Position auch im Parlament durchzusetzen; die Oppositionsfraktionen werden das Instrument nutzen, um sich auf dem Feld der nationalen Europapolitik gegen die Regierung zu profilieren. Im Extremfall könnte die nationale Politisierung der europäischen Subsidiaritätskontrolle zu einem reduzierten Einfluss der nationalen Parlamente auf den europäischen Gesetzgebungsprozess und zu verringerten Spielräumen gegenüber der Exekutive führen.

4. Wenn das Instrument der Subsidiaritätsrüge von den nationalen Parlamenten vornehmlich als Instrument der Ablehnung eines europäischen Legislativvorschlags verstanden und genutzt werden wird, dann wird sich über kurz oder lang die Frage stellen, ob die bürokratische und aufwendige europarechtliche Prüfung eines Vorschlags entlang des Subsidiaritätsgrundsatzes überhaupt erforderlich ist, oder ob eine Abfrage der Europäischen Kommission zur politischen Wünschbarkeit und Durchsetzbarkeit eines Vorschlags in den nationalen Parlamenten nicht ausreichend wäre.

\section{Literatur}

Ausschuss der Regionen, 2012, Subsidiaritätsbericht 2011, https://portal.cor.europ a.eu/subsidiarity (Stand: 21.7.2013).

Becker, Peter/Pintz, Anne, 2012: Die neue Rolle der nationalen Parlamente in der EU. Aus deutschen und europäischen Zeitschriften und Gutachten der Jahre 2010 bis 2012, SWP-Zeitschriftenschau, 2012/ZS 03, Berlin.

Becker, Peter/Kietz, Daniela, 2010: Zwischen Brüssel, Berlin und Karlsruhe: Bundestag und Bundesrat als Vorzeigemodell parlamentarischer Mitwirkung in der Europapolitik?, Heinrich-Böll-Stiftung, Berlin, http://www.boell.de/downloads /parlamentarischemitwrikungineuropa.pdf (Stand: 21.7.2013).

Börzel, Tanja, 2000: Europäisierung und innerstaatlicher Wandel. Zentralisierung und Entparlamentarisierung in: PVS 41 (2), 225-250.

O'Brennan, John/Raunio, Tapio (Hrsg.), 2007: National Parliaments within the Enlarged European Union: From Victims of Integration to Competitive Actors, London. 
Brok, Elmar/Selmayr, Martin, 2008: Der ,Vertrag der Parlamente“ als Gefahr für die Demokratie? Zu den offensichtlich unbegründeten Verfassungsklagen gegen den Vertrag von Lissabon, in: integration 31 (3), 217-234.

Buschmann, Marco/Daiber, Birgit, 2011: Subsidiaritätsrüge und Grundsatz der begrenzten Einzelermächtigung«, in: DÖV 64 (13), 504.510.

Bundesrat, 2010: Beschluss des Bundesrates zur Initiative des Königreichs Belgien, der Republik Bulgarien, der Republik Estland, des Königreichs Spanien, der Französischen Republik, der Italienischen Republik, der Republik Ungarn, der Republik Polen, der Portugiesischen Republik, Rumäniens, der Republik Finnland und des Königreichs Schweden für eine Richtlinie des Europäischen Parlaments und des Rates über die europäische Schutzanordnung PE-CONS 2/10, Drs. 43/10 (Beschluss) vom 26. März 2010.

Bundesrat, 2012: Vorschlag für eine Verordnung des Rates über die Ausübung des Rechts auf Durchführung kollektiver Maßnahmen im Kontext der Niederlassungs- und der Dienstleistungsfreiheit, Drs. 158/12 Beschluss vom 15.6.2012.

Buzogány, Aron/Stuchlik, Andrej, 2012: Subsidiarität und Mitsprache. Nationale Parlamente nach Lissabon, in: ZParl 43 (2), 340-361.

Cooper, Ian, 2006: The Watchdogs of Subsidiarity: National Parliaments and the Logic of Arguing in the EU, in: JCMS 44 (2), 281-304.

Cooper, Ian, 2012: A ,Virtual Third Chamber" for the European Union? National Parliaments after the Treaty of Lisbon, in: West European Politics 35 (3), 441-465.

COSAC, 2011, Sixteenth Bi-annual Report: Developments in European Union. Procedures and Practices Relevant to Parliamentary Scrutiny, October 2011, http:// www.cosac.eu/documents/bi-annual-reports-of-cosac (Stand: 21.7.2013).

COSAC, 2012, Eighteenth Bi-annual Report: Developments in European Union.

Procedures and Practices Relevant to Parliamentary Scrutiny, October 2012, http://www.cosac.eu/documents/bi-annual-reports-of-cosac (Stand: 21.7.2013). Craig, Paul, 2012: Subsidiarity: A Political and Legal Analysis, in: JCMS 50 (1), 72-87.

Cygan, Adam, 2011: The Parliamentarisation of the EU Decision-Making? The Impact of the Treaty of Lisbon on National Parliaments, in: European Law Review 36, 480-499.

Cygan, Adam, 2012: National Parliaments within the EU-polity - no longer loosers but hardly victorious, in: ERA Forum 12 (4), 517-533.

Deutscher Bundestag, 2012: Referat PA 1, Kurzmitteilung aus Brüssel, Nr. 1/2012 vom 25. Mai 2012. 
Die Subsidiaritätsprüfung in Bundestag und Bundesrat

Europäische Kommission, 2012 a: Impact Assessment Board Report for 2011, SEC (2012) 101 fin., Brüssel 1.2.2012.

Europäische Kommission, 2012 b: Vorschlag für eine Verordnung des Rates über die Ausübung des Rechts auf Durchführung kollektiver Maßnahmen im Kontext der Niederlassungs- und der Dienstleistungsfreiheit, KOM (2012) 130 endg. vom 21.3.2012.

Europäische Kommission, 2012 c: Bericht über die Anwendung der Grundsätze der Subsidiarität und der Verhältnismäßigkeit (19. Bericht über „Bessere Rechtsetzung“" 2011), KOM (2012)373 endg., Brüssel, den 10.7.2012.

Europäische Kommission, 2012 d: Jahresbericht 2011 über die Beziehungen zwischen der Europäischen Kommission und den nationalen Parlamenten, KOM (2012)375 endg., Brüssel, den 10.7.2012.

Europäische Kommission, 2013 a: Impact Assessment Board Report for 2012, http://ec.europa.eu/governance/impact/key_docs/docs/iab_report_2012_en_final.pdf (Stand: 21.7.2013).

Europäische Kommission, 2013 b: Report from the Commission, Annual Report 2012 on Relations between the European Commission and National Parliaments, COM (2013)565 fin., Brussels, 30.7.2013.

Europäischer Konvent, 2003: Entwurf der Verfassung, Band I- Überarbeiteter Text von Teil I, CONV 724/03, Brüssel, den 24. Mai 2003.

Gröning-von Thüna, Sebastian, 2010: Die neuen Begleitgesetze zum Vertrag von Lissabon aus Sicht des Deutschen Bundestages - offene Fragen und neue Herausforderungen, in: integration 33 (4), 313-333.

Hölscheidt, Sven, 2008: Formale Aufwertung - geringe Schubkraft: die Rolle der nationalen Parlamente gemäß dem Lissabonner Vertrag, in: integration 31 (3), 254-265.

Horsley, Thomas, 2012: Subsidiarity and the European Court of Justice: Missing Pieces in the Subsidiarity Jigsaw, in: JCMS 50 (2), 267-282.

Kiiver, Philipp, 2012: The Conduct of Subsidiarity Checks of EU Legislative Proposals by National Parliaments: Analysis, Observations und Practical Recommendations «, in: ERA Forum 12 (4), 535-547.

Kiiver, Philipp, 2011: The Early-Warning System for the Principle of Subsidiarity: The National Parliament as a Conseil d'Etat for Europe, in: European Law Review 36 (1), 98-108.

Koch, Jessica/Kullas, Matthias, 2010: Subsidiarität nach Lissabon - Scharfes Schwert oder stumpfe Klinge?, Centrum für Europäische Politik (CEP-Studie) März 2010, Freiburg i. Br. 
Kropp, Sabine/Buzogány, Aron/Buche, Jonas, 2012: Von den Schwierigkeiten, Zusammengehöriges zu vereinen - Nationale Parlamente und Exekutiven als Gegenstand der Europäisierungsforschung, in: PVS 53 (1), 109-134.

Lorz, Ralph Alexander, 2010: Wissenschaftliche Stellungnahme zum öffentlichen Expertengespräch „Prüfung des unionsrechtlichen Subsidiaritätsprinzips“ im Unterausschuss Europarecht des Rechtsauschusses des Deutschen Bundestages am 16. Juni 2010, http://www.bundestag.de/bundestag/ausschuesse17/a06/ua europarecht/anhoerungen/01_Subsidiaritaet/02_Stellungnahmen/Stellungnahm e_Prof_Lorz.pdf (Stand: 21.7.2013).

Mayer, Franz C., 2009: Der EuGH als Feind? Die Debatte um das soziale Europa in der europäischen Rechtsprechung, in: integration 32 (3), 246-265.

Mayer, Franz C., 2010: Stellungnahme zum öffentlichen Expertengespräch „Prüfung des unionsrechtlichen Subsidiaritätsprinzips“ im Unterausschuss Europarecht des Rechtsauschusses des Deutschen Bundestages am 16. Juni 2010, http:// www.bundestag.de/bundestag/ausschuesse17/a06/ua_europarecht/anhoerunge n/01_Subsidiaritaet/02_Stellungnahmen/Stellungnahme_Mayer.pdf (Stand: 21.7.2013).

Norton, Philipp (Hrsg.), 1996: National Parliaments and the European Union, London.

Raunio, Tapio, 2011: The Gatekeepers of the European Integration? The Functions of National Parliaments in the EU Political System, in: Journal of European Integration 33 (3), 303-321.

Raunio, Tapio, 2009: National Parliaments and the European Integration: What We Know and Agenda for Future Research: in: The Journal of legislative Studies 15 (4), 317-334.

Raunio, Tapio/Hix, Simon, 2000: Backbenchers learn to fight back: European integration and parliamentary government, in: West European Politics 23 (4), 142-168.

Schröder, Hinrich, 2012: Die Mitwirkung des Bundestages in EU-Angelegenheiten nach dem EUZBBG in der Praxis - ein Kurzkommentar, in: ZfParl 2/2012, 250-277.

Semmler, Jörg, 2010: Die Subsidiaritätsrüge nach dem Vertrag von Lissabon - Plädoyer für ein Politisches Instrument, in: ZEuS 13 (4), 529-538.

Suhr, Oliver, 2010: Stellungnahme zum öffentlichen Expertengespräch „Prüfung des unionsrechtlichen Subsidiaritätsprinzips" im Unterausschuss Europarecht des Rechtsauschusses des Deutschen Bundestages am 16. Juni 2010, http://ww w.bundestag.de/bundestag/ausschuesse17/a06/ua_europarecht/anhoerungen/01 
Subsidiaritaet/02_Stellungnahmen/Stellungnahme_Suhr.pdf (Stand: 21.7.2013).

Uerpmann-Wittzack, Robert, 2009: Frühwarnsystem und Subsidiaritätsklage im deutschen Verfassungssystem, in: EuGRZ 36 (17-20), 461-468.

Korrespondenzanschrift:

Dr. Peter Becker

Stiftung Wissenschaft und Politik (SWP)

Ludwigkirchplatz 3-4

10719 Berlin

E-Mail: peter.becker@swp-berlin.org 